\title{
SCONES: Self-Consistent Neural Network for Protein Stability Prediction Upon Mutation
}

\author{
Yashas Samaga B L, Shampa Raghunathan, U. Deva Priyakumar \\ Submitted date: 04/06/2021 - Posted date: 04/06/2021
}

Licence: CC BY-NC-ND 4.0

Citation information: Samaga B L, Yashas; Raghunathan, Shampa; Priyakumar, U. Deva (2021): SCONES: Self-Consistent Neural Network for Protein Stability Prediction Upon Mutation. ChemRxiv. Preprint. https://doi.org/10.26434/chemrxiv.14729445.v1

Engineering proteins to have desired properties by mutating amino acids at specific sites is commonplace. Such engineered proteins must be stable to function. Experimental methods used to determine stability at throughputs required to scan the protein sequence space thoroughly are laborious. To this end, many machine learning based methods have been developed to predict thermodynamic stability changes upon mutation. These methods have been evaluated for symmetric consistency by testing with hypothetical reverse mutations. In this work, we propose transitive data augmentation, evaluating transitive consistency, and a new machine learning based method, first of its kind, that incorporates both symmetric and transitive properties into the architecture. Our method, called SCONES, is an interpretable neural network that estimates a residue's contributions towards protein stability dG in its local structural environment. The difference between independently predicted contributions of the reference and mutant residues in a missense mutation is reported as dG. We show that this self-consistent machine learning architecture is immune to many common biases in datasets, relies less on data than existing methods, and is robust to overfitting.

File list (2)

scones_maintext.pdf (2.33 MiB)

view on ChemRxiv - download file 


\title{
SCONES: Self-Consistent Neural Network for Protein Stability Prediction Upon Mutation
}

\author{
Yashas Samaga B L, Shampa Raghunathan, and U. Deva Priyakumar* \\ Center for Computational Natural Sciences and Bioinformatics, International Institute of \\ Information Technology, Hyderabad 500 032, India \\ E-mail: deva@iiit.ac.in
}

\begin{abstract}
Engineering proteins to have desired properties by mutating amino acids at specific sites is commonplace. Such engineered proteins must be stable to function. Experimental methods used to determine stability at throughputs required to scan the protein sequence space thoroughly are laborious. To this end, many machine learning based methods have been developed to predict thermodynamic stability changes upon mutation. These methods have been evaluated for symmetric consistency by testing with hypothetical reverse mutations. In this work, we propose transitive data augmentation, evaluating transitive consistency, and a new machine learning based method, first of its kind, that incorporates both symmetric and transitive properties into the architecture. Our method, called SCONES, is an interpretable neural network that estimates a residue's contributions towards protein stability $(\Delta G)$ in its local structural environment. The difference between independently predicted contributions of the reference and mutant residues in a missense mutation is reported as $\Delta \Delta G$. We show that this self-consistent machine learning architecture is immune to many common biases in datasets, relies less on data than existing methods, and is robust to overfitting.
\end{abstract}




\section{Introduction}

Proteins are large biomolecules that naturally evolved to perform specific biological functions.1 One of the exciting advances in recent decades has been the ability to design and develop new synthetic proteins with desired properties. ${ }^{2}$ 5 Synthetic proteins have a myriad of applications ranging from vaccine development ${ }^{6]}$ to tackling environmental problems. $\frac{[17}{5.7}$ Biotechnology and biomedical industries use engineered proteins that achieve superior functions. ${ }^{8] 12}$ For example, engineered enzymes react faster and are more efficient. ${ }^{13[14}$ Protein engineering is a promising field and has vast academic and industrial interests.

One approach to protein engineering is redesigning existing proteins to have desired properties. $\frac{15[16}{16}$ Natural proteins are not necessarily optimal; $\frac{17}{17}$ moreover, physiological conditions in which proteins naturally evolved are often different from industrial and laboratory conditions. $\frac{15}{}$ Proteins are therefore mutated to optimize their properties, and one such property is protein stability. $\frac{15|18| 19}{}$ Any protein must be stable in its environmental conditions for it to be useful. 20 Proteins are mutated to increase stability and often to compensate for destabilization arising from other functionally important mutations. ${ }^{21}$ However, the protein sequence space is overwhelmingly large. Experimentally determining the stability of many variants is laborious and expensive, $\frac{13222}{21}$ and in-silco simulations required (e.g., molecular dynamics free energy calculations) to accurately predict stability with high throughput are computationally prohibitive. ${ }^{23 \mid 24}$ To narrow down the search space for experimental validation, fast computational methods have been developed to predict protein stability changes upon mutation. $\sqrt[25] 38]{38}$ These methods either classify a mutation as stabilizing or destabilizing, or predict a real-valued target indicating the extent of stabilization or destabilization. Popular real-valued targets are: change in denaturation midpoint temperature, $\stackrel{3940}{ }$ denoted by $\Delta T_{m}$; change in thermal deactivation temperature,, 40 denoted by $\Delta T_{50}$; and change in free energy of folding,,$^{25|27| 28 \mid 33}$ denoted by $\Delta \Delta G . T_{m}, T_{50}$ and $\Delta G$ are state functions of proteins. $\Delta T_{m}, \Delta T_{50}$ and $\Delta \Delta G$ are computed by taking the difference between their respective state values in the reference and mutant proteins. This definition confers useful properties that 
can be exploited to evaluate and improve the performance of protein stability predictors. We present the properties for $\Delta \Delta G$, but they are also applicable to $\Delta T_{m}$ and $\Delta T_{50}$.

A protein must exist in its folded form to function. The stability of a protein refers to its ability to exist in its folded form relative to its denatured state. $\frac{4142}{42}$ The thermodynamic stability of a protein can thus be quantified by the difference in the Gibbs free energy between the unfolded and folded state, denoted by $\Delta G$ :

$$
\Delta G=G_{\text {unfolded }}-G_{\text {folded }}
$$

The change in stability upon mutation can be quantified by the change in $\Delta G$, denoted by $\Delta \Delta G$.

$$
\Delta \Delta=\Delta G_{\text {mutant }}-\Delta G_{\text {reference }}
$$

One of the properties of $\Delta \Delta G$ is anti-symmetry. The $\Delta \Delta G$ for a hypothetical reverse mutation must be equal in magnitude but opposite in sign to that of the corresponding forward mutation in identical experimental conditions.

$$
\Delta \Delta G_{\text {forward }}=\Delta G_{2}-\Delta G_{1}=-\left(\Delta G_{1}-\Delta G_{2}\right)=-\Delta \Delta G_{\text {reverse }}
$$

Another property that $\Delta \Delta G$ confers is transitivity: given three proteins $X, Y$ and $Z$, the $\Delta \Delta G$ of a mutation that goes from $X \rightarrow Z$ is the sum of the $\Delta \Delta G$ s of $X \rightarrow Y$ and $Y \rightarrow Z$ mutations provided the experimental conditions are kept the same throughout.

$$
\begin{gathered}
\Delta \Delta G_{X \rightarrow Z}=\Delta G_{Z}-\Delta G_{X} \\
=\left(\Delta G_{Z}-\Delta G_{Y}\right)+\left(\Delta G_{Y}-\Delta G_{X}\right) \\
=\Delta \Delta G_{Y \rightarrow Z}+\Delta \Delta G_{X \rightarrow Y}
\end{gathered}
$$


The transitive property can be repeatedly applied to form long chains of mutations. The mutations in a chain can occur at different sites: we can start with two point-mutation samples and end up with a double-mutation sample. We can restrict the mutation site to a fixed position while constructing transitive pairs if we require that the resulting combined mutation be a point-mutation.

A variety of computational methods have been developed to predict protein stability changes upon mutation. There are methods based on molecular dynamics, 2324 physicsbased force fields, ${ }^{47}$ empirical and statistical potentials ${ }^{[28148}$ and more recently machine learning..$^{251273614950 \mid}$ ThermoNet ${ }^{27}$ uses an ensemble of 3D Convolutional Neural Networks (CNN) on voxelized mutation site environments to predict $\Delta \Delta G$. PremPS ${ }^{\sqrt{25}}$ uses Random Forest (RF) regression on ten evolutionary and structure-based features to predict $\Delta \Delta G$. The use of machine learning to solve problems in fundamental sciences has surged in recent

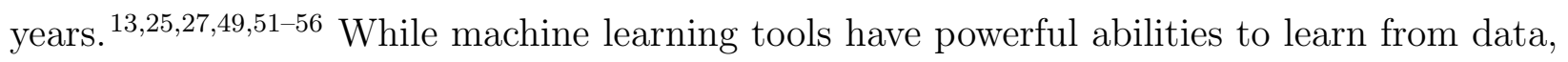
their power also makes them prone to learning noise and spurious correlations, especially when training data is biased and limited. Methods that predict protein stability changes upon mutation must exhibit symmetric and transitive properties to be consistent. Existing methods have been evaluated for symmetric consistency, and many older methods were shown to lack consistency ${ }^{43} 46657$ Symmetric inconsistencies in earlier methods were mainly resolved by augmenting the training set with hypothetical reverse mutations. ${ }^{252738}$ Few methods have incorporated symmetric consistency directly into their model architecture. ${ }^{45158}$ As far as we know, no method has used a transitively augmented training set or checked for transitive consistency (Section 1 in Supporting Information). We present a neural-network-based method called Self-COnsistent NEural network protein Stability prediction (SCONES) that incorporates both symmetric and transitive properties of $\Delta \Delta G$ into the architecture. The neural network predicts the contributions of a residue towards the protein's $\Delta G$. $\Delta G$ contributions are predicted for the reference and mutant residues of a missense mutation independently, and $\Delta \Delta G$ is calculated by taking the difference between the predicted $\Delta G$ s. These architec- 
tural constraints for self-consistency help regularize models at the cost of ease of learning. We demonstrate that this self-consistent architecture is robust to overfitting and can predict protein stability changes upon mutation well.

\section{Methods}

Our method is a neural network that estimates a residue's contributions towards protein stability in its local structural environment. For a missense mutation, the difference between the independently predicted contributions of the reference and mutant residues is reported as $\Delta \Delta G$. Section 2.1 describes the architecture in detail. The method is trained using datasets derived from FireProtDB ${ }^{[59}$ and evaluated on $\mathrm{S} 350, \stackrel{31}{\mathbf{S}^{\text {sym }}[55}$ and $\mathrm{S} 767^{26}$ test sets. Section 2.2 elaborates on training set construction and evaluation datasets. We use an ensemble of fifty models obtained from ten rounds of five-fold cross-validation for evaluation. Section 2.3 describes the training procedure in detail.

\subsection{Architecture}

Our method is a single neural network that estimates a residue's contributions in its local structural environment to the protein's $\Delta G$. We predict $\Delta \Delta G$ for a missense mutation by taking the difference between the predicted $\Delta G$ contributions of the reference and mutant residues. This architecture incorporates both symmetric and transitive properties of $\Delta \Delta G$.

The local environment of a residue (referred to as the central residue henceforth) is defined to consist of all neighboring residues whose $C_{\beta}$ atom is within $8 \AA$ distance from the $C_{\beta}$ atom of the central residue (Figure 1). The local environment can be thought of as a graph with residues as nodes and interactions as edges. Our neural network operates on each edge (residue-residue interaction) independently and estimates its contributions towards $\Delta G$. The

sum of all contributions of a residue's interactions with its neighbors is predicted as its total contribution towards protein stability (Figure 2 and Figure 3). The node features consist 
of residue chemical descriptors and solvent accessible surface area (features are listed in Table 1). We do not provide a one-hot encoding of the amino acids as it increases the odds of overfitting in some datasets (Section 2 in Supporting Information). The edge features

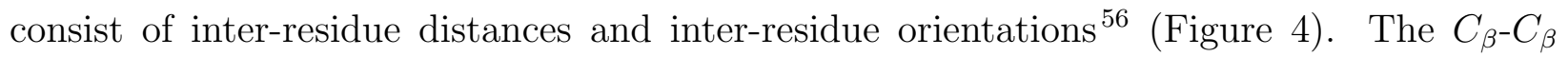
distance (referred to as $d$ henceforth) of any two residues is the distance between the $C_{\beta}$ atoms of the two residues. We use Glycine's $C_{\alpha}$ atom in place of $C_{\beta}$ atom for computing $d$. $d$ raised to various powers and exponentials of $d$ (which appear in theoretical formulations of pair potentials) are given as inter-residue distance features. The inter-residue orientation angles are defined in Figure 4. The sine and cosine of the angles are provided as interresidue orientation features. Orientation features requiring Glycine's $C_{\beta}$ atom are set to zero. We use the reference protein's structure to compute the structure-based features for both reference and mutant proteins. This ensures that the same set of neighbors in the local environment are considered while calculating $\Delta G$ contributions of the central residue in the reference and mutant proteins. We do not use any features that involve both reference and mutant residues (such as the change in residue volume) explicitly to maintain the consistency properties of the architecture. The two residues' node features and the edge features form the network's input features. See Figure S1 and Section 3 in Supporting Information for detailed information on the architecture.

Table 1: List of node features

\begin{tabular}{l|l|}
\hline Feature & Description or AAIndex $^{\mathrm{a}}$ \\
\hline Formal Charge & Table S5 in Supporting Information \\
Normalized Van der Waals volume & FAUJ880103 \\
Hydropathy index & KYTJ820101 \\
Steric parameter & CHAM810101 \\
Polarity & GRAR740102 \\
Residue accessible surface area in tripeptide & CHOC760101 \\
Solvent Accessible Surface Area & calculated using DSSP $60 \mid 61$ \\
aAIndex ${ }^{62}$ is a database of numerical indices for physicochemical and biochemical \\
properties of amino acids and pairs of amino acids. The code in the column is the \\
AAIndex database ID of the property.
\end{tabular}




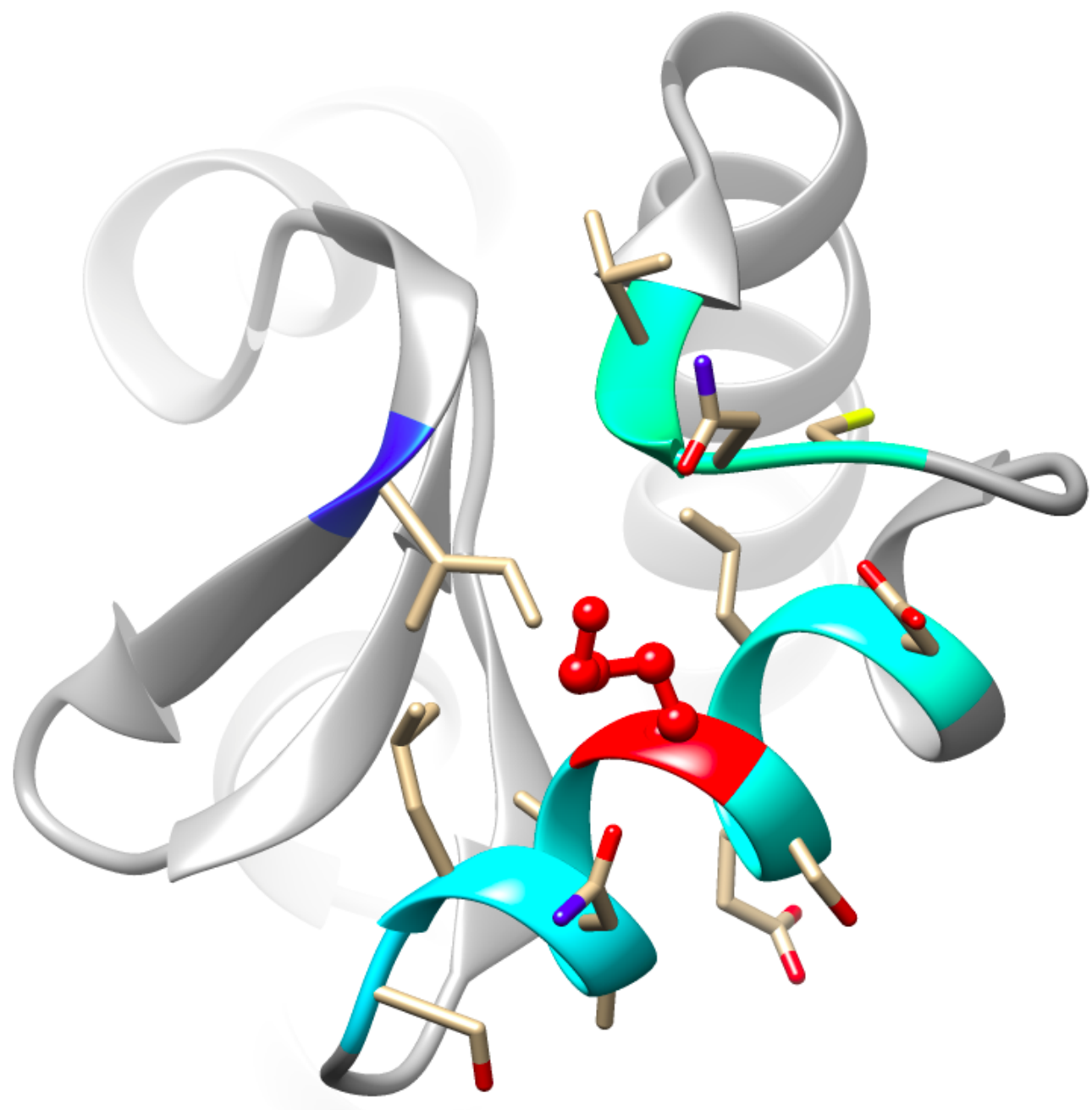

Figure 1: Residue Local Environment The red-colored residue in ball and stick representation is the central residue. The highlighted residues with side-chain atoms shown with sticks are the neighboring residues. 

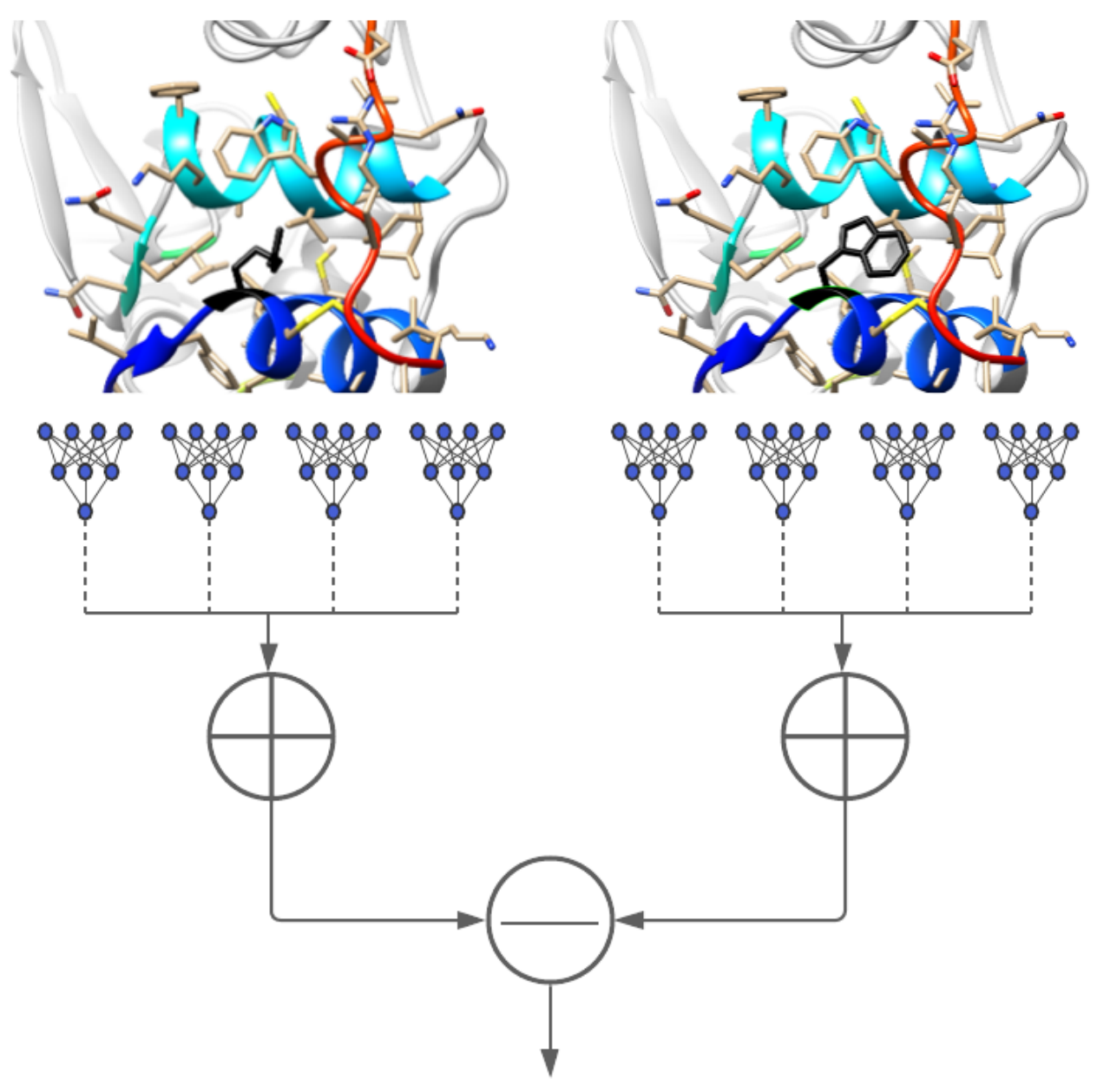

$\Delta \Delta G$

Figure 2: Self-Consistent Architecture The neural network estimates the contributions of a residue pair's interactions towards $\Delta G$ in the local environment. These estimates for all central-residue neighbor-residue pairs are added to predict the total $\Delta G$ contribution of the central residue. The same procedure is applied on both reference and mutant proteins to obtain two $\Delta G$ estimates. The difference between the two is the predicted $\Delta \Delta G$. 


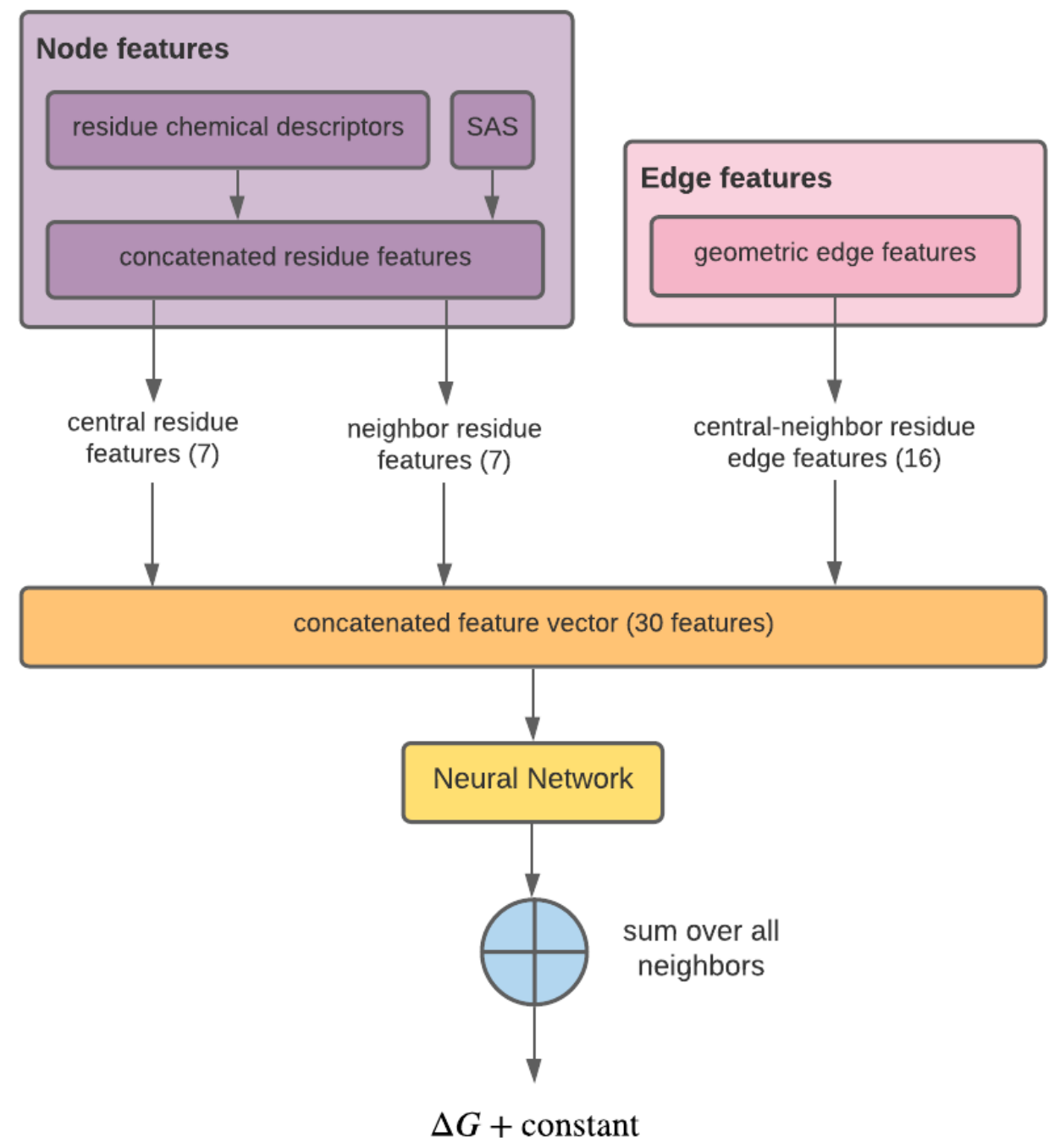

Figure 3: Overview of the model The neural network estimates the contributions of a residue pair's interactions towards $\Delta G$. The two residues' node features and edge features are concatenated to form the network's input feature vector. For a given local environment, feature vectors are constructed for all central-residue neighbor-residue pairs. The network predictions for all the neighbors are then summed over to predict the $\Delta G$ contributions of the central residue. The constant factor contains contributions to the protein's $\Delta G$ that are not accounted for in our calculations. We assume such contributions to be identical in both reference and mutant proteins. It is fixed for a given protein and local environment, and it cancels out when we calculate $\Delta \Delta G$. SAS stands for Solvent Accessible Surface area. 

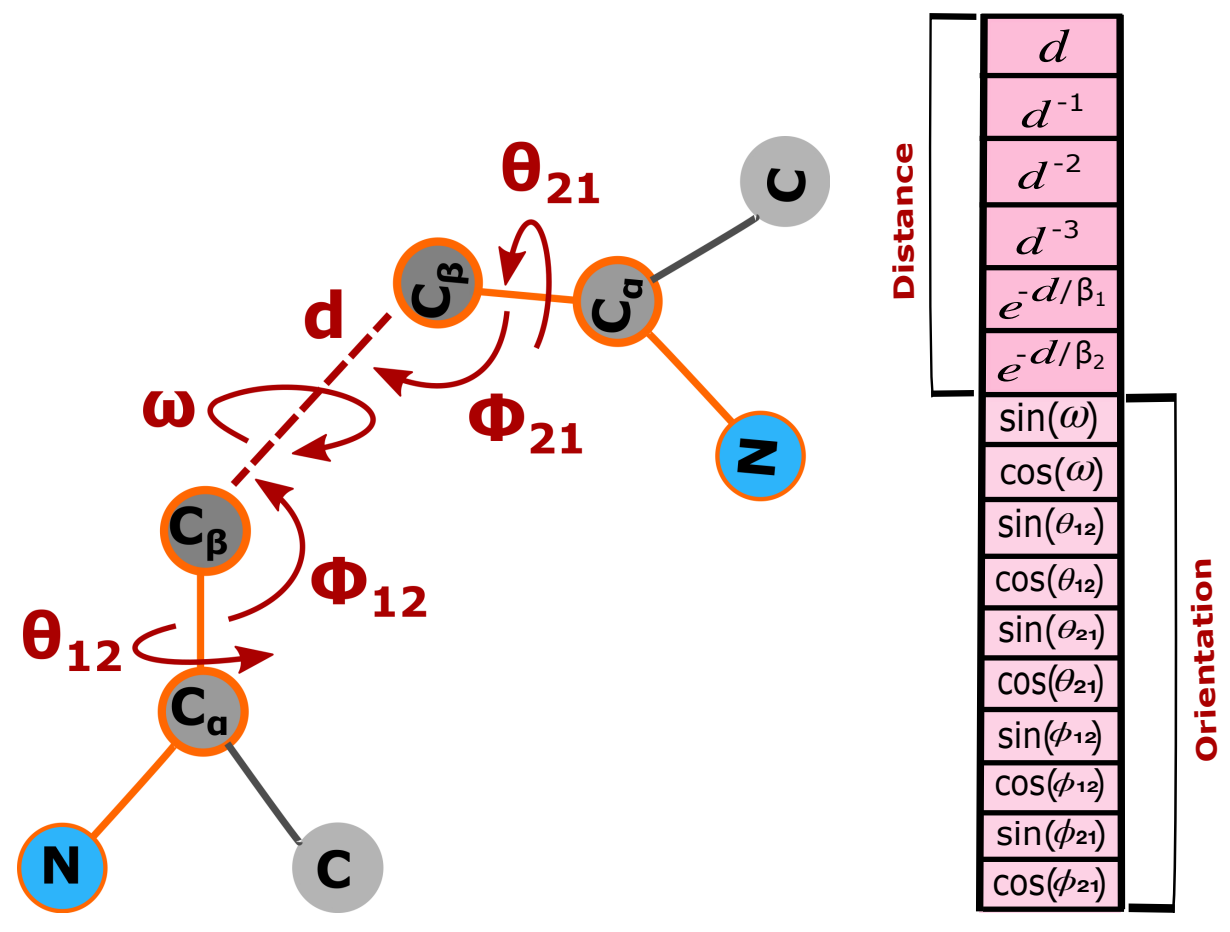

Figure 4: Visualization of geometric edge features between two residues $\theta$ and $\phi$ depend on the choice of the first residue. $d$ and $\omega$ are the same for both residues irrespective of the residue order. The two planar angles $\left(\phi_{12}\right.$ and $\left.\phi_{21}\right)$, three dihedral angles $\left(\omega, \theta_{12}\right.$ and $\left.\theta_{21}\right)$ and $d$ fully describe the relative positions of the backbone atoms. ${ }^{56}$ All the distancederived features are normalized to have zero mean and unit standard deviation. Note that these features capture the backbone conformation but not the side-chain conformation. 
The self-consistency constraints on the architecture help regularize the models. A prediction for hypothetical reverse mutation would be equivalent to predicting and subtracting the $\Delta G$ estimates in the reverse order, which would yield an exactly negated prediction of $\Delta \Delta G$ of the forward mutation unless different structures are used. Regression loss functions generally used are functions of the absolute difference between the predicted and target value. Therefore, losses for forward and their corresponding reverse mutations are the same, and augmenting the training set with hypothetical reverse mutations is the same as duplicating the entire dataset. Our architecture also captures the transitive property, but transitive samples can still aid in learning, unlike hypothetical reverse mutations. Losses for $\Delta \Delta G_{X \rightarrow Y}$, $\Delta \Delta G_{Y \rightarrow Z}$ and $\Delta \Delta G_{X \rightarrow Z}$ are different and not linear combinations of each other due to the non-linearity of loss functions. Therefore, new transitive samples can give substantially different learning signals (Section 1.1 in Supporting Information). The network learns to predict $\Delta G$ indirectly from $\Delta \Delta G$ reference dataset. This makes learning difficult: the optimization process needs to learn to estimate the two $\Delta G$ values from the $\Delta \Delta G$ target and then further estimate individual contributions to each $\Delta G$ value (Figure 2). There are over ten neighbors in a typical sample; that would mean the method needs to separate around twenty components of a sum from the $\Delta \Delta G$ target. We show that the model can still learn to predict protein stability changes without requiring explicit supervision at a finer level.

\subsection{Dataset}

FireProtDB ${ }^{59}$ is a database of point-mutations which have experimental thermostability data. We curated a new dataset from FireProtDB according to the following criteria:

- Samples with structures where the mutated residue or its neighboring residues did not have $N, C_{\alpha}$, and $C_{\beta}$ atom coordinates $\left(C_{\beta}\right.$ atom coordinates is not required for Glycine) were removed.

- Samples with known pH that lie outside two and twelve were removed. The samples 
with unknown $\mathrm{pH}$ were assumed to lie in the range under the assumption that the benefits of having more samples outweigh the cost of having a few outlier samples.

- Samples with $|\Delta \Delta G|$ greater than $8 \mathrm{kcal} / \mathrm{mol}$ were removed.

- A sample is a duplicate of another if the reference and mutant proteins involved are identical in both samples and the absolute difference in the $\mathrm{pH}$ of the two samples is less than 0.1. $\Delta \Delta G$ values of duplicate samples were merged into one sample by averaging. The entire group of duplicates was discarded if the standard deviation of the $\Delta \Delta G$ values exceeded $0.5 \mathrm{kcal} / \mathrm{mol}$.

The resulting curated dataset contains 5989 samples.

The data available is scarce, and it may not be feasible to create a single test set that is balanced on all accounts. Hence, we used multiple test sets, with each used to evaluate a different aspect of the method. We evaluated our method on S350, $\mathbf{S}^{\text {sym } 45}$ and S767 datasets. The S350 dataset has 54 stabilizing samples $(\Delta \Delta G>0.5 \mathrm{kcal} / \mathrm{mol}), 107$ neutral samples $(|\Delta \Delta G| \leq 0.5 \mathrm{kcal} / \mathrm{mol})$, and 189 destabilizing samples $(\Delta \Delta G<-0.5 \mathrm{kcal} / \mathrm{mol})$. Our method treats stabilizing and destabilizing mutations identically, and hence S350 can be used to evaluate the performance. $\mathbf{S}^{\mathrm{sym}}$ is a dataset consisting of samples for which both reference and mutant structures are available. This test set is used to evaluate the symmetric consistency in methods. The composition of both S350 and $\mathbf{S}^{\text {sym }}$ test sets are not balanced across mutation types; both test sets are abundant in mutations involving hydrophobic residues like alanine and valine but lack sufficient samples in other categories. Therefore, we also evaluate on a category-wise balanced dataset created by Frenz et al. ${ }^{26}$ which we call S767.

Two training sets of varying difficulty were created for each test set from the curated dataset after removing samples similar to the test samples. Two samples are similar if they satisfy all of the following conditions:

- The samples have the same mutation or the reverse mutation of each other. 
- An 11-residue amino acid sequence centered around the mutating residue is extracted for both samples, and the sequence identity of the extracted sequences is greater than $50 \%$.

The first training set was created based on the above similarity criteria. This training set contains samples at the same mutation site but with a different mutation as some samples in the test sets. The effects of different mutations in the same local environment may not be independent. There can be a mutation site where mutations always cause minor changes in protein stability irrespective of the mutation type. In such a situation, the model can learn to output a near-zero stability change for that site irrespective of the mutation type. Test data can leak into the training set this way which would result in overestimation of performance. Therefore, we created a more challenging training set by filtering with the second condition alone. We refer to the first kind of training sets as "easy" training sets and the second kind as "hard" training sets. We omit "easy" in future references for the first kind of training sets, and hard training sets will have "hard" explicitly mentioned.

The training set for $\mathbf{S}^{\text {sym }}$ might contain transitive samples that can be obtained from $\mathbf{S}^{\text {sym }}$ or samples that can be used to create transitive samples which are a part of $\mathbf{S}^{\text {sym }}$. Hence, for an unbiased evaluation, we also evaluate the performance on $\mathbf{S}^{\mathbf{s y m}}$ dataset after filtering the training set against the transitive closure of $\mathbf{S}^{\mathrm{sym}}$ (which we call $\mathbf{S}^{\text {transitive }}$ in future references). The filtered training sets for both $\mathbf{S}^{\text {sym }}$ and $\mathbf{S}^{\text {transitive }}$ are almost the same. The hard training sets are identical, and the easy training sets are similar (Table 2); this suggests that our filtering protocol is good. We also use the transitive pairs in $\mathbf{S}^{\text {transitive }}$ to evaluate the transitive consistency of our method.

\subsection{Training}

Bioinformatics related processing was carried out using BioPython. ${ }^{64}$ The amino acid chemical descriptors were obtained from AAIndex database ${ }^{\sqrt{62}}$ using the quantiprot ${ }^{65}$ python package. The neural network was trained using PyTorch,,$\sqrt[66]{ }$ and evaluation metrics were computed 
Table 2: List of test sets

\begin{tabular}{|c|c|c|c|}
\hline Dataset & Actual Size ${ }^{a}$ & Filtered Size ${ }^{b}$ & Description \\
\hline $\mathrm{S} 350$ & 350 & 345 & $\begin{array}{l}\text { This test set was created by randomly sam- } \\
\text { pling mutations from } \mathrm{S} 2648^{3163}\end{array}$ \\
\hline S87 & 87 & 86 & $\begin{array}{l}\text { This is a subset of S350 for which }|\Delta \Delta G| \text { ex- } \\
\text { ceeds } 2 \mathrm{kcal} / \mathrm{mol} \text {. }\end{array}$ \\
\hline $\mathbf{S}^{\mathrm{sym}}$ & 684 & 683 & $\begin{array}{l}\text { This test set consists of samples which have } \\
\text { both reference and mutant structures. It con- } \\
\text { tains both forward and reverse mutations. }\end{array}$ \\
\hline $\mathbf{S}^{\text {transitive c }}$ & 1601 & 1596 & $\begin{array}{l}\text { This test set is the transitive closure of all sam- } \\
\text { ples in } \mathbf{S}^{\text {sym }} \text {. Nine hundred seventeen transi- } \\
\text { tive pairs can be formed from the samples. All } \\
\text { the samples have a reference structure. }\end{array}$ \\
\hline S767 & 767 & 745 & $\begin{array}{l}\text { This dataset is a mutation category-wise bal- } \\
\text { anced dataset created to evaluate performance } \\
\text { on different mutation types and identify po- } \\
\text { tential biases in methods. }\end{array}$ \\
\hline
\end{tabular}

a The size of the originally published dataset.

$\mathrm{b}$ The size of the dataset after removing samples that had incomplete backbone structural information for residues in the mutation site's local environment.

${ }^{\mathrm{c}} \mathbf{S}^{\text {transitive }}$ is a new test set introduced in this work. The actual size indicates the number of samples after computing the transitive closure on $\mathbf{S}^{\text {sym }}$. The filtered size is the number of samples that have complete backbone structural information for the residues in the mutation site's local environment.

Table 3: List of training sets

\begin{tabular}{|ll|}
\hline Dataset $^{\text {a }}$ & Size \\
\hline S350 & 5532 \\
S350 (hard) & 2735 \\
S $^{\text {sym }}$ & 5290 \\
S $^{\text {sym }}$ (hard) & 2654 \\
S $^{\text {transitive }}$ & 5267 \\
S $^{\text {transitive (hard) }}$ & 2654 \\
S767 & 4857 \\
S767 (hard) & 1176 \\
\hline
\end{tabular}

a The name indicates the test set based on which the training set was filtered. The filtering protocol is specified in parenthesis for "hard" protocol and omitted for "easy" protocol. 
using scikit-learn. ${ }^{67}$ PyTorch and NumPy ${ }^{68}$ were used for general array processing.

The loss function (denoted by $\mathcal{L}$ ) consists of general adaptive robust loss function by

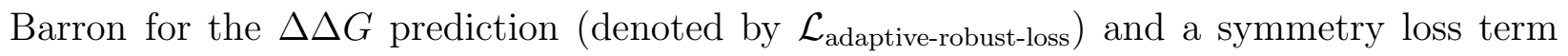
(denoted by $\mathcal{L}_{\text {symmetry-loss }}$ ). The symmetry loss enforces the requirement that the interaction energy between two residues must be identical irrespective of the order of the residues in the feature vector. Feature vectors for both residue orders are generated during training, and the mean value is returned as the $\Delta G$ contribution. The $\mathcal{L}_{\text {symmetry-loss }}$ ensures that the predictions from both feature vectors are equal.

$$
\begin{gathered}
\mathcal{L}=\mathcal{L}_{\text {adaptive-robust-loss }}+\alpha \mathcal{L}_{\text {symmetry-loss }} \\
\mathcal{L}_{\text {symmetry-loss }}=\frac{1}{N} \sum_{i} \sum_{j}\left(E_{c j}-E_{j c}\right)^{2}
\end{gathered}
$$

where $\alpha$ is a scalar hyperparameter, $c$ is the index of the central residue, $j$ iterates over the indices of the neighboring residues, $E_{c j}$ and $E_{j c}$ are the energies predicted for residue $c$ and $j$ in the two possible orders, $i$ iterates over the minibatch and $N$ is the minibatch size.

Adam optimizer ${ }^{70}$ was used with a weight decay of 0.004 and default parameters for the rest. The initial learning rate was set to 0.002 and decreased by a factor of ten every time the learning plateaued (Section 2 in Supporting Information for detailed training procedure). The model was trained for forty epochs with a minibatch size of thirty-two. The models were trained using five-fold cross-validation with the training set. The model with the minimum validation loss was saved as the final model for each training instance. The final prediction is the ensemble average of all predictions from the models generated in ten rounds of crossvalidation (fifty models in total). This process is repeated for each training set. All the code may be obtained from the authors. 


\section{Results and Discussion}

We evaluated the performance of our method on S350, $\mathbf{S}^{\text {sym }}$ and S767 test sets. We created two separate training sets of varying difficulty for each test set after removing similar samples (Section 2.2). For each test set, the performance metrics were computed from the average ensemble predictions of all the models from ten rounds of cross-validation. Table 4 reports the regression performance on all test sets. Our method provides balanced performance for both stabilizing and destabilizing mutations across all test sets. The variation in performance across test sets can be attributed to their vastly different compositions. In S350, one-third of the mutations involve alanine, and about half of the mutations involve alanine or valine. $\mathbf{S}^{\text {sym }}$ and S767 also suffer from similar biases but to a lesser extent (Figures S2, S3, and S4 in Supporting Information). Table S6 in Supporting Information provides category-wise performance statistics for S767 test set. Figure 5 shows the Receiver Operating Characteristic (ROC) curve for strongly stabilizing and destabilizing mutations computed from ensemble predictions for $\mathbf{S}^{\text {sym }}$ test set. The curves show that our method is capable of identifying significantly stabilizing and destabilizing mutations equally well.
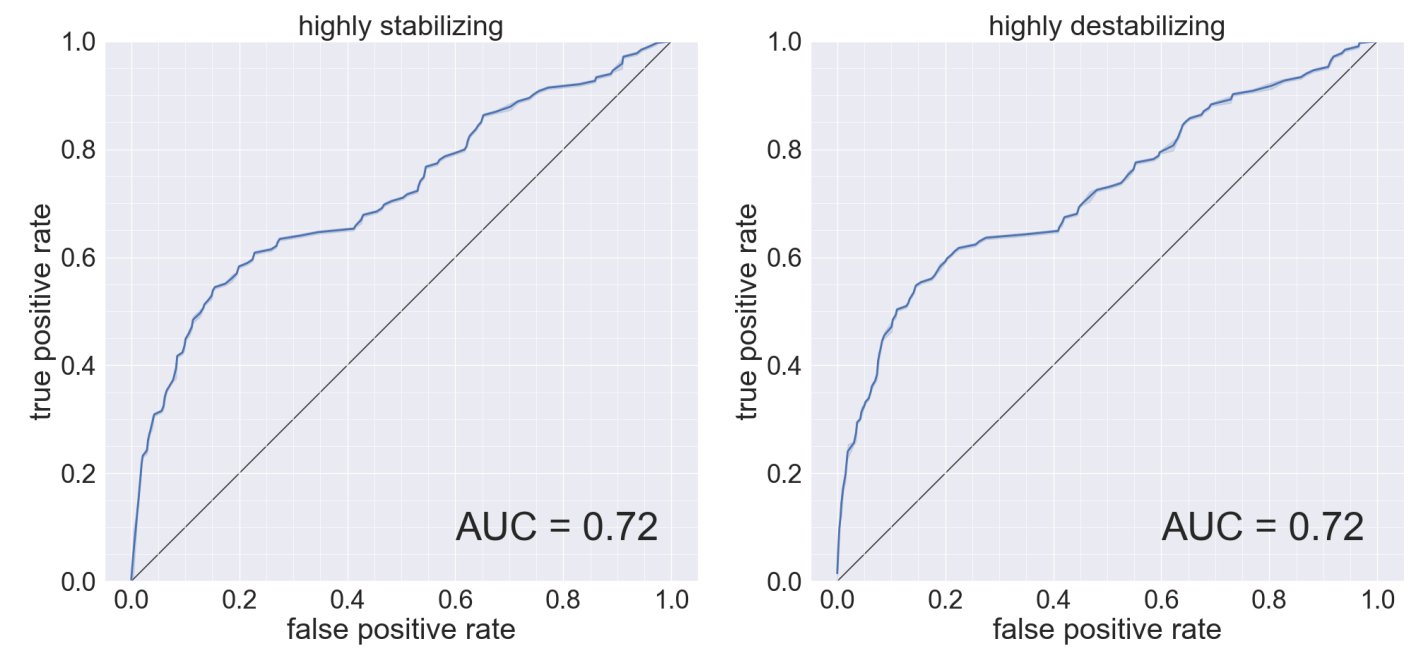

Figure 5: ROC plots for classifying strongly stabilizing and destabilizing mutations in $\mathrm{S}^{\mathrm{sym}}$ with predictions obtained from ensemble average of the models trained using $\mathbf{S}^{\text {sym }}$ (hard) Left: ROC plot for strongly stabilizing mutations $(\Delta \Delta G>1 \mathrm{kcal} / \mathrm{mol})$. Right: ROC plot for strongly destabilizing mutations $(\Delta \Delta G<-1 \mathrm{kcal} / \mathrm{mol})$. 
Table 4: Performance on different test sets

\begin{tabular}{|c|c|c|c|c|c|c|c|c|}
\hline \multirow[b]{2}{*}{ training set } & \multirow[b]{2}{*}{ test set } & \multirow[b]{2}{*}{$\mathrm{PCC}$} & \multirow[b]{2}{*}{$\mathrm{MAE}^{\mathrm{a}}$} & \multirow[b]{2}{*}{$\mathrm{RMSE}^{\mathrm{a}}$} & \multicolumn{2}{|c|}{ stabilization } & \multicolumn{2}{|c|}{ destabilization } \\
\hline & & & & & $\mathrm{AUC}^{\mathrm{b}}$ & $\mathrm{PCC}^{\mathrm{C}}$ & $\mathrm{AUC}^{\mathrm{b}}$ & $\mathrm{PCC}^{\mathrm{C}}$ \\
\hline S350 & S87 & 0.67 & 1.75 & 2.14 & 0.95 & 0.22 & 0.95 & 0.24 \\
\hline S350 (hard) & S87 & 0.66 & 1.85 & 2.21 & 0.94 & 0.11 & 0.94 & 0.25 \\
\hline $\mathrm{S} 350$ & S350 & 0.56 & 1.13 & 1.52 & 0.81 & 0.62 & 0.75 & 0.47 \\
\hline S350 (hard) & S350 & 0.53 & 1.17 & 1.54 & 0.79 & 0.57 & 0.73 & 0.45 \\
\hline $\mathbf{S}^{\mathrm{sym}}$ & $\mathbf{S}^{\mathrm{sym}}$ & 0.50 & 1.13 & 1.61 & 0.72 & 0.34 & 0.73 & 0.43 \\
\hline $\mathbf{S}^{\text {transitive }}$ & $\mathbf{S}^{\mathrm{sym}}$ & 0.50 & 1.13 & 1.61 & 0.71 & 0.34 & 0.73 & 0.43 \\
\hline $\mathbf{S}^{\text {sym }}$ (hard) & $\mathrm{S}^{\mathrm{sym}}$ & 0.48 & 1.16 & 1.63 & 0.72 & 0.34 & 0.72 & 0.38 \\
\hline S767 & S767 & 0.40 & 1.30 & 1.98 & 0.67 & 0.33 & 0.66 & 0.35 \\
\hline S767 (hard) & S767 & 0.37 & 1.37 & 2.03 & 0.61 & 0.09 & 0.64 & 0.36 \\
\hline
\end{tabular}

PCC stands for Pearson's Correlation Coefficient, AUC stands for Area Under ROC Curve, RMSE stands for Root Mean Squared Error, and MAE stands for Mean Absolute Error.

${ }^{a}$ RMSE and MAE are in $\mathrm{kcal} / \mathrm{mol}$.

${ }^{\mathrm{b}}$ AUC is calculated for strongly affecting mutations only $(|\Delta \Delta G| \geq 1 \mathrm{kcal} / \mathrm{mol})$.

${ }^{\mathrm{c}} \mathrm{PCC}$ is calculated using all the samples.

Table 5 compares the performance of our method on S350 with existing methods. Many methods that are biased towards predicting destabilizing mutations perform well on S350 but fare poorly on balanced test sets like $\mathbf{S}^{\text {sym }}$ (Table 6). Amongst the methods compared in both Table 5 and Table 6, only SCONES and PremPS show balanced performance on both S350 and $\mathbf{S}^{\text {sym }}$ test sets; all other methods show poor symmetric consistency with $\mathbf{S}^{\text {sym }}$.

The prediction for forward mutations and their corresponding reverse mutations in identical experimental conditions must be strongly negatively correlated. Many older methods show a poor negative correlation. $\underline{43} \underline{46157744}$ The poor performance is largely due to the methods inheriting the biases of unbalanced training sets that contained far more destabilizing mutations than stabilizing mutations. The $\mathbf{S}^{\text {sym }}$ dataset consists of mutations for which both reference and mutant structures are available and is used to evaluate the symmetric consistency in methods. Table 6 compares the performance of our method on $\mathbf{S}^{\mathrm{sym}}$ with existing methods. The symmetric consistency has been traditionally measured using a correlation coefficient $R_{F R}$ and mean prediction bias $\langle\delta\rangle . \underline{46} R_{F R}$ is the correlation coefficient between the predictions for forward mutations and their corresponding reverse mutations. The $\delta$ for 
Table 5: Comparison of performance on S350

\begin{tabular}{|c|c|c|c|c|}
\hline \multirow[b]{2}{*}{ Method } & \multicolumn{2}{|c|}{ S350 } & \multicolumn{2}{|c|}{ S87 } \\
\hline & $\mathrm{PCC}$ & $\mathrm{RMSE}^{\mathrm{a}}$ & $\mathrm{PCC}$ & $\mathrm{RMSE}^{\mathrm{a}}$ \\
\hline SCONES $^{b}$ & 0.56 & 1.52 & 0.67 & 2.13 \\
\hline SCONES $\left(\right.$ hard) ${ }^{c}$ & 0.53 & 1.54 & 0.66 & 2.21 \\
\hline PremPS & 0.72 & 1.09 & 0.81 & 1.52 \\
\hline PremPS ${ }^{\mathrm{P} 25}$ & 0.58 & 1.28 & 0.60 & 1.94 \\
\hline $\mathrm{mCSM}^{36}$ & 0.73 & 1.08 & 0.82 & 1.48 \\
\hline MAESTRO 34 & 0.70 & 1.13 & 0.76 & 1.67 \\
\hline PoPMuSiC v2.0 & 0.67 & 1.16 & 0.71 & 1.67 \\
\hline PoPMUSiC v1.071 & 0.62 & 1.24 & 0.70 & 1.66 \\
\hline $\mathrm{SDM} 2{ }^{48}$ & 0.61 & 1.29 & 0.69 & 1.71 \\
\hline $\mathrm{SDM}^{35}$ & 0.52 & 1.80 & 0.63 & 2.11 \\
\hline Dmutant & 0.48 & 1.81 & 0.57 & 2.31 \\
\hline AUTOMUTE ${ }^{72}$ & 0.46 & 1.43 & 0.45 & 1.99 \\
\hline CUPSAT 73 & 0.37 & 1.91 & 0.50 & 2.14 \\
\hline
\end{tabular}

Our method only accepted 345 samples out of 350 . Data was taken from Refs. $\frac{25 \mid 31 / 34 / 36 / 48}{\text { The training }}$ sets of all methods did not have samples from S350. PremPS $^{\mathrm{P}}$ did not have homologous proteins in the training set.

a RMSE is in $\mathrm{kcal} / \mathrm{mol}$.

b Trained using S350 (easy) training set.

c Trained using S350 (hard) training set. 
a sample is defined as $\Delta \Delta G_{\text {forward }}+\Delta \Delta G_{\text {reverse }}$ and the average $\delta$ over all samples (denoted by $\langle\delta\rangle)$ is used as a measure of overall consistency of the method. However, this metric is prone to hiding symmetric biases in both directions: negative errors can cancel out positive errors. Therefore, we computed the root mean squared error of the prediction biases $\left(\sqrt{\left\langle\delta^{2}\right\rangle}\right.$, denoted by $\Delta$ ). A method with perfect symmetric consistency will have an $R_{F R}$ value of negative one, $\langle\delta\rangle$ value of zero, and $\Delta$ value of zero. Table 6 and Figure 6 show that our method has excellent symmetric consistency. Our method has a near-perfect $R_{F R}$ value and a low $\Delta$ value.
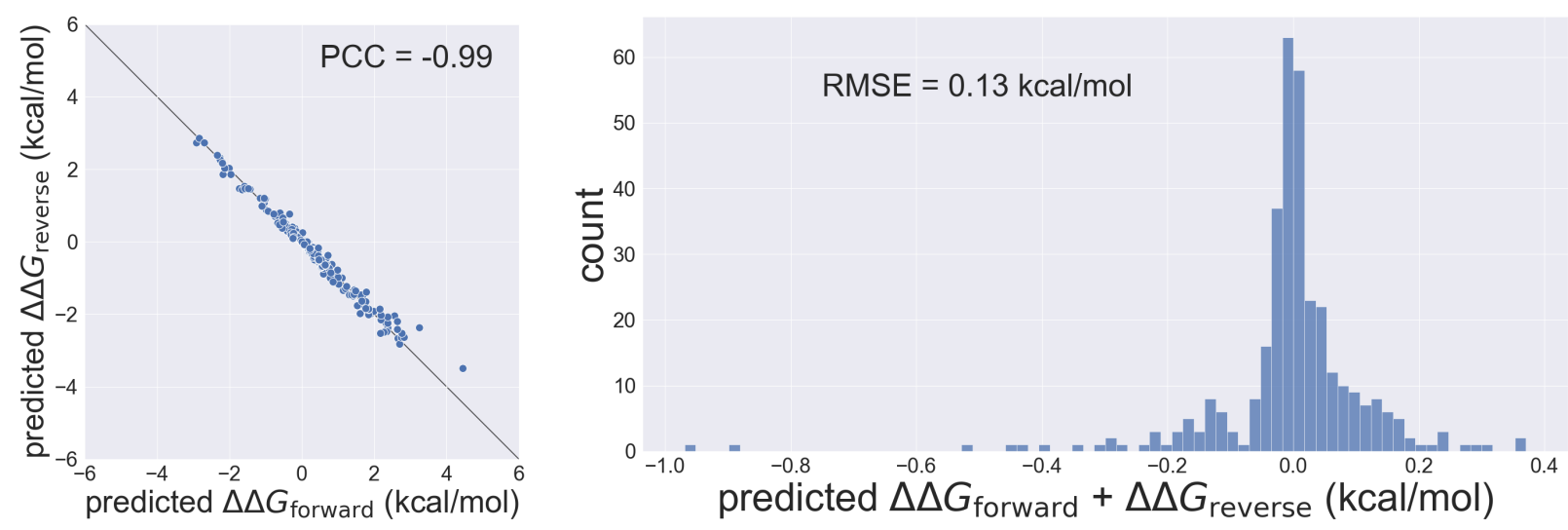

Figure 6: The mean predictions of an ensemble consisting of all the models from all the rounds of cross-validation trained using $\mathbf{S}^{\text {sym }}$ (hard) training set is used. Left: plot of predicted values of $\Delta \Delta G$ for forward and reverse mutations. Right: distribution of prediction bias $\left(\Delta \Delta G_{\text {forward }}+\Delta \Delta G_{\text {reverse }}\right)$

Table 7 presents transitive consistency metrics computed using $\mathbf{S}^{\text {transitive }}$. There are 917 transitive pairs amongst the 1601 samples in $\mathbf{S}^{\text {transitive }}$. We introduce two new metrics to evaluate transitive consistency: correlation coefficient $R_{T}$ and norm $\Delta_{T} . R_{T}$ is the correlation between $\Delta \Delta G_{X \rightarrow Y}+\Delta \Delta G_{Y \rightarrow Z}$ and $\Delta \Delta G_{X \rightarrow Z}$, and $\Delta_{T}$ is the root mean squared value of $\Delta \Delta G_{X \rightarrow Y}+\Delta \Delta G_{Y \rightarrow Z}-\Delta \Delta G_{X \rightarrow Z}$ computed over all samples. A method with perfect transitive consistency must have an $R_{T}$ of one and $\Delta_{T}$ of zero. Table 7 and Figure 7 show that our method has very good transitive consistency.

The models trained with the hard training set show better consistency properties but lower prediction performance than the models trained with the easy training set. This 
Table 6: Comparison of performance on $\mathrm{S}^{\text {sym }}$

\begin{tabular}{|c|c|c|c|c|c|c|c|}
\hline \multirow[b]{2}{*}{ Method } & \multicolumn{2}{|c|}{ forward } & \multicolumn{2}{|c|}{ reverse } & \multirow[b]{2}{*}{$R_{F R}$} & \multirow[b]{2}{*}{$\langle\delta\rangle^{\mathrm{a}}$} & \multirow[b]{2}{*}{$\Delta^{\mathrm{a}}$} \\
\hline & PCC & RMSE & PCC & $\mathrm{RMSE}^{\mathrm{a}}$ & & & \\
\hline SCONES $^{b}$ & 0.50 & 1.58 & 0.46 & 1.64 & -0.95 & 0.07 & 0.33 \\
\hline SCONES $\left(\mathbf{S}^{\text {transitive }}\right)^{c}$ & 0.50 & 1.58 & 0.46 & 1.64 & -0.95 & 0.07 & 0.33 \\
\hline SCONES $(\text { hard })^{\mathrm{d}}$ & 0.45 & 1.63 & 0.45 & 1.63 & -0.99 & 0.00 & 0.13 \\
\hline PremPS & 0.64 & 1.21 & 0.56 & 1.30 & -0.91 & 0.03 & 0.34 \\
\hline PremPS ${ }^{\mathrm{P}} 25$ & 0.56 & 1.32 & 0.50 & 1.37 & -0.89 & 0.04 & 0.33 \\
\hline ThermoNet*27 & 0.58 & 1.38 & 0.59 & 1.38 & -0.95 & -0.05 & - \\
\hline ThermoNet ${ }^{27}$ & 0.47 & 1.56 & 0.47 & 1.55 & -0.96 & -0.01 & 0.23 \\
\hline PoPMuSiC ${ }^{\text {sym } 45]}$ & 0.48 & 1.58 & 0.48 & 1.62 & -0.77 & 0.03 & - \\
\hline PremPS ${ }^{25}$ & 0.81 & 0.96 & 0.74 & 1.12 & -0.93 & 0.03 & 0.38 \\
\hline DDGun3D ${ }^{28}$ & 0.56 & 1.42 & 0.53 & 1.46 & -0.99 & -0.02 & 0.14 \\
\hline INPS ${ }^{[32]}$ & 0.51 & 1.42 & 0.50 & 1.44 & -0.99 & -0.04 & - \\
\hline DDGun $^{[28}$ & 0.48 & 1.47 & 0.48 & 1.50 & -0.99 & -0.01 & 0.04 \\
\hline INPS3D $^{33}$ & 0.59 & 1.29 & 0.44 & 1.64 & -0.86 & -0.55 & - \\
\hline Rosetta & 0.69 & 2.31 & 0.43 & 2.61 & -0.41 & -0.69 & - \\
\hline FoldX ${ }^{[30}$ & 0.63 & 1.56 & 0.39 & 2.13 & -0.38 & -0.47 & - \\
\hline MAESTRO ${ }^{34}$ & 0.52 & 1.36 & 0.32 & 2.09 & -0.34 & -0.58 & - \\
\hline $\mathrm{SDM}^{35]}$ & 0.51 & 1.74 & 0.32 & 2.28 & -0.75 & -0.32 & - \\
\hline PoPMuSiC v2.129 & 0.63 & 1.21 & 0.25 & 2.18 & -0.29 & -0.71 & - \\
\hline $\mathrm{mCSM}^{36]}$ & 0.61 & 1.23 & 0.14 & 2.43 & -0.26 & -0.91 & - \\
\hline DUET $^{37}$ & 0.63 & 1.20 & 0.13 & 2.38 & -0.21 & -0.84 & - \\
\hline MUPRO & 0.79 & 0.94 & 0.07 & 2.51 & -0.02 & -0.97 & - \\
\hline
\end{tabular}

SCONES, PremPS ${ }^{\mathrm{M}}$ and PremPSP ${ }^{\mathrm{P}}$, ThermoNet, ThermoNet* and PoPMuSiC ${ }^{\text {sym }}$ did not have overlapping samples in their training set. ThermoNet and PremPS ${ }^{\mathrm{P}}$ also removed homologous proteins from their training set. All other methods were evaluated directly on the test set. Data was taken from Chen et al., Li et al., Pucci et al.

${ }^{a} \mathrm{RMSE},\langle\delta\rangle$ and $\Delta$ are in $\mathrm{kcal} / \mathrm{mol}$.

b Trained using $\mathbf{S}^{\text {sym }}$ (easy) training set.

${ }^{c}$ Trained using $\mathbf{S}^{\text {transitive }}$ (easy) training set.

d Trained using $\mathbf{S}^{\text {sym }}$ (hard) training set.

Table 7: Evaluating transitive consistency on $\mathbf{S}^{\text {transitive }} . \Delta_{T}$ is in $\mathrm{kcal} / \mathrm{mol}$.

\begin{tabular}{|c|c|c|}
\hline Method & $R_{T}$ & $\Delta_{T}{ }^{\mathrm{a}}$ \\
\hline SCONES $\left(\mathbf{S}^{\mathrm{sym}}\right)$ & 0.97 & 0.24 \\
\hline SCONES $\left(\mathbf{S}^{\text {transitive }}\right)$ & 0.97 & 0.24 \\
\hline SCONES ( $\mathbf{S}^{\text {sym }}$ hard $)$ & 0.99 & 0.13 \\
\hline
\end{tabular}

${ }^{\mathrm{a}} \Delta_{T}$ is in $\mathrm{kcal} / \mathrm{mol}$. 

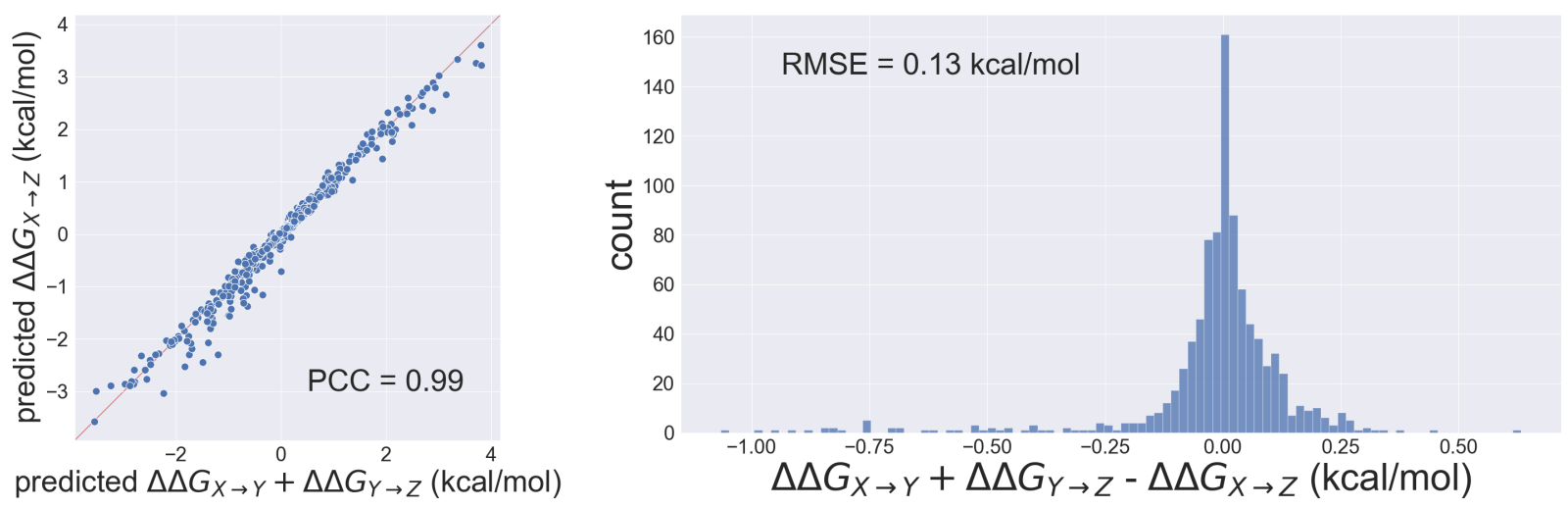

Figure 7: The mean predictions of an ensemble consisting of all the models from all the rounds of cross-validation trained with $\mathbf{S}^{\text {sym }}$ (hard) training set is used. Left: plot of $\Delta \Delta G_{X \rightarrow Y}+$ $\Delta \Delta G_{Y \rightarrow Z}$ vs $\Delta \Delta G_{X \rightarrow Z}$. Right: distribution of prediction bias $\left(\Delta \Delta G_{X \rightarrow Y}+\Delta \Delta G_{Y \rightarrow Z}-\right.$ $\left.\Delta \Delta G_{X \rightarrow Z}\right)$

indicates a potential trade-off between consistency and prediction performance. It could also mean that the easy unbalanced training sets lead to overfitting, resulting in poor consistency properties while showing high prediction performance. On the other extreme, a method that always outputs a constant zero will have perfect consistency properties but would not give helpful predictions. Therefore, it is essential to conduct a holistic evaluation of a method using many test sets and evaluation metrics. Overall, our method gives reliable predictions while also being self-consistent.

Figure 8 shows the variation in performance of trained models in each training set. We note that there is significant variance. The neural network has to learn to predict dozens of components of $\Delta \Delta G$ from limited training data without supervision at $\Delta G$ or interaction energy level. We believe that the variance can be reduced by improved sampling procedures for training sets and using waning auxiliary losses from force fields to guide the optimization at a finer level. The average performance on a test set, however, does not vary significantly between easy and hard training sets (Table 4). This suggests that our method is robust to overfitting.

The nature of computations in our method is hierarchical. At the lowest level of the hierarchy, our neural network predicts $\Delta G$ contributions of individual residue-residue in- 


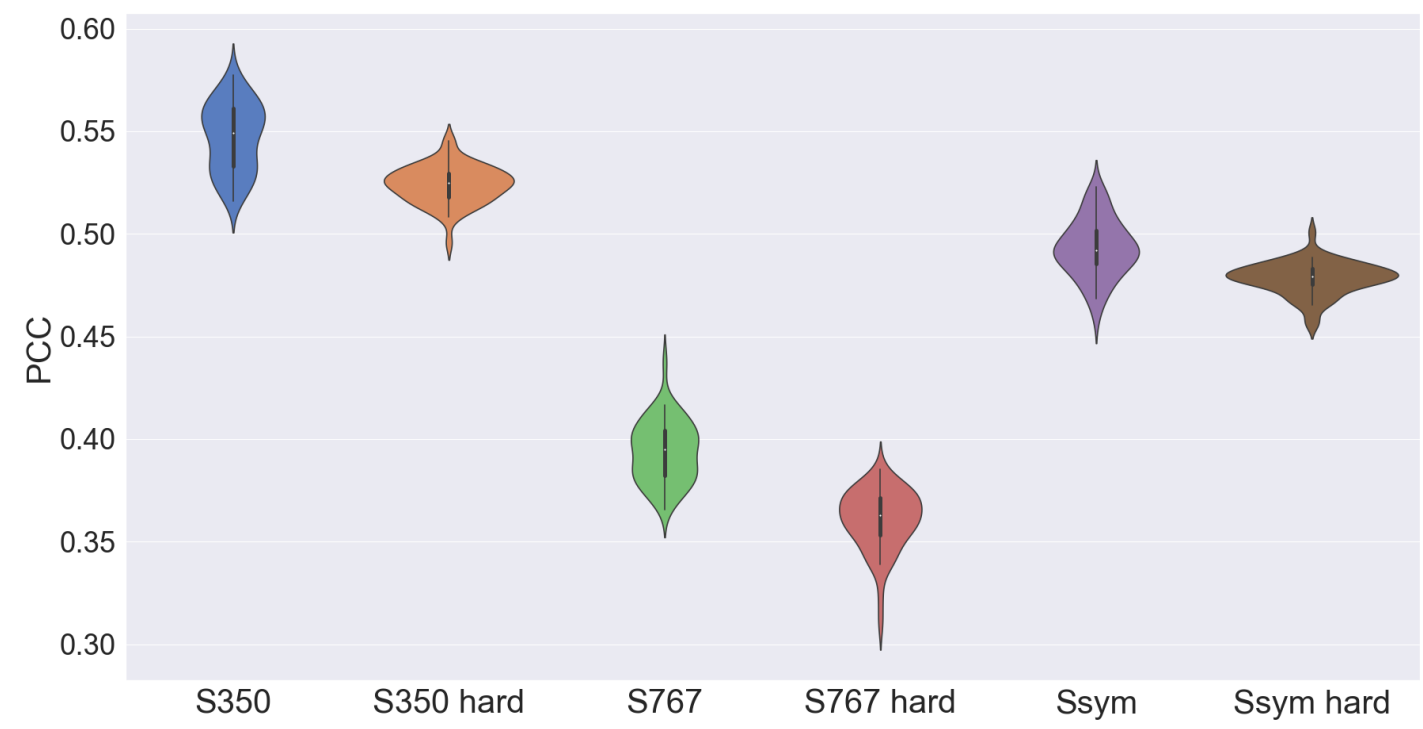

Figure 8: Variance in model performance Pearson Correlation Coefficient (PCC) was computed for each of the fifty models from ten rounds of cross-validation.

teractions. These predictions can provide valuable insights into causes of stabilization or destabilization. An interaction map can summarize these intermediate predictions. Such interaction maps have been previously used to chemically interpret solvation free energies of druglike molecules in organic solvents. $\frac{5176}{}$ Here, the interaction map consists of two columns representing the reference and mutant residues, and several rows representing neighboring residues. Each cell contains the $\Delta G$ contributions predicted for the interactions between the central-residue (column) and the neighboring residue (row). We used the ensemble average of the intermediate predictions to plot the interaction map in Figure 9. The interaction map suggests that the mutation leads to better beneficial hydrophobic interactions which result in extra stabilization.

We showed that our highly constrained self-consistent architecture could perform well on standard test sets. The method shows balanced performance on stabilizing and destabilizing mutations. The variation in performance between easy and hard test sets is minimal, which indicates robustness. The architectural constraints significantly reduce overfitting based on random patterns in mutation types or sequence similarity. Our architecture is hierarchical 


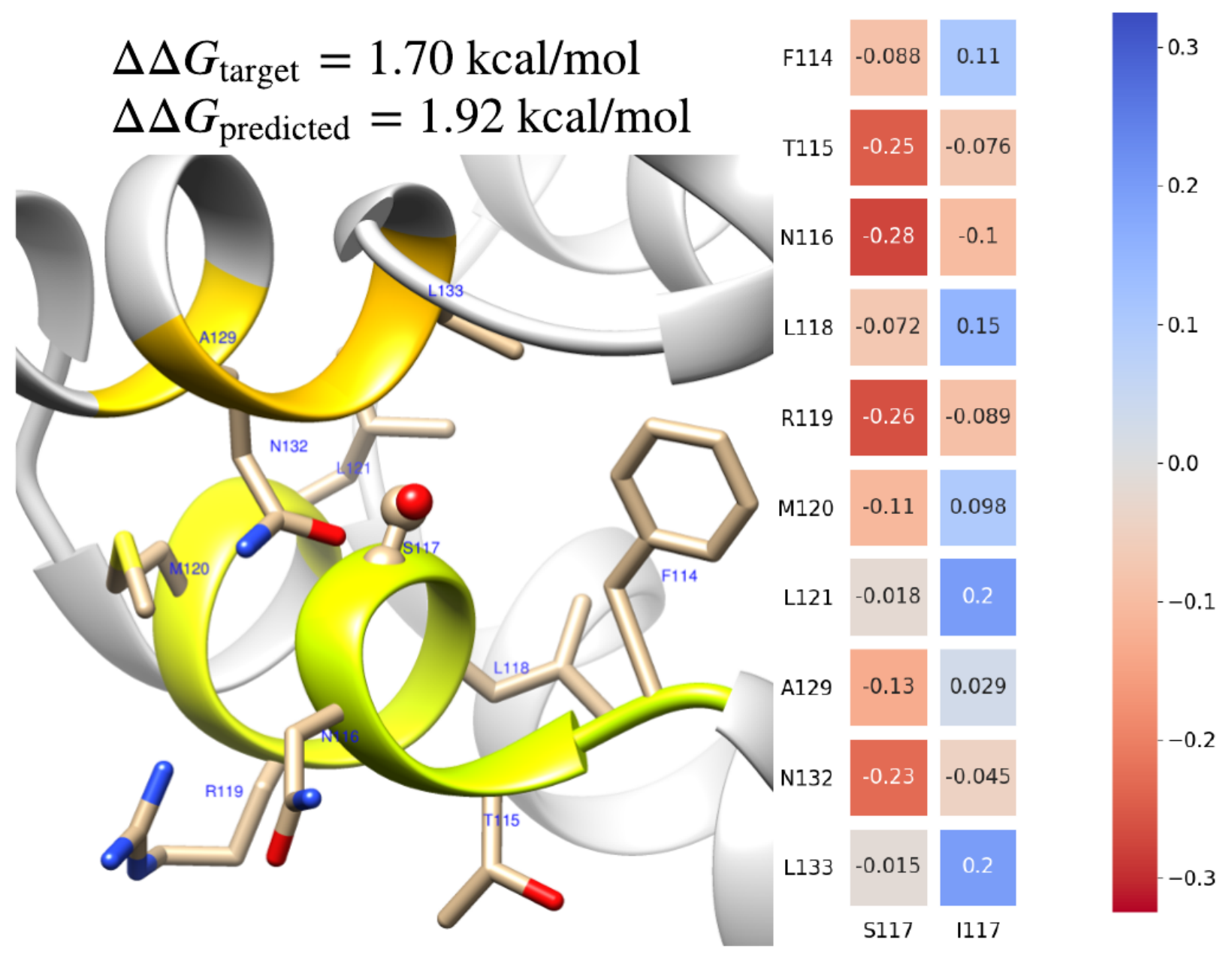

Figure 9: Interaction map for serine to isoleucine mutation in bacteriophage T4 lysozyme (pdb id: 2lzm). Isoleucine provides new beneficial hydrophobic interactions that lead to improved protein stability. The ensemble average of the intermediate predictions from the models trained using S767 (hard) dataset was used to plot the interaction map. 
and is similar to force field computations which allows easy integration of auxiliary losses from force fields. Work along this is in progress. The nature of computation also provides high interpretability to the method. We directly use structures without any minimization or backbone relaxation protocols that were previously demonstrated to improve the performance. $\frac{25 / 27 / 30 / 77 / 78}{1}$ We proposed a new architecture and showed its viability. We hope that further research will find the ideas introduced in this work useful and build on this architecture.

\section{Conclusion}

Protein engineering is a rapidly growing field with vast potential for biotechnological and biomedical applications. Protein stability is an important property that has to be tuned in protein engineering. Additionally, optimization of other properties of proteins should be done such that the proteins are still stable. Many computational methods have been developed to identify potential stability-altering mutations. The amount of data available to train protein stability predictors upon mutation is small. Incorporating domain knowledge directly into the method can help overcome dataset limitations. Commonly used regression targets confer many properties which have not been fully exploited; the transitive property has been feebly used in existing methods. Here, we have proposed improving performance by incorporating the transitive property into model architectures and augmenting training sets with transitive samples. We have presented a method that incorporates both symmetric and transitive properties of $\Delta \Delta G$ into the architecture. It consists of a neural network that predicts a residue's contributions towards the protein's $\Delta G$. The difference in the independently

predicted $\Delta G$ contributions for the reference and mutant residues in a missense mutation is returned as $\Delta \Delta G$. This method relies less on the data to learn self-consistency properties and is immune to many common problems arising from unbalanced and undersampled datasets. Our method does not use any features that involve both reference and mutant proteins. This, 
along with the independence in calculations of each $\Delta G$ contribution, makes it very robust towards overfitting. Our method is simple but belongs to a class of methods that has not been explored thoroughly. The method can be extended to use more complex architectures such as graph neural networks and incorporate more domain knowledge using the method's hierarchical architecture. We hope that further exploration in this direction and the use of ideas presented in this work will help improve protein stability predictors.

\section{Acknowledgement}

We thank IHub-Data, IIIT Hyderabad and DST/WOS-A (grant, no. SR/WOS-A/CS19/2018 (G)) for the financial support. We acknowledge Devdarshni Priyakumar for useful suggestions.

\section{Supporting Information Available}

Training procedure details, detailed neural network architecture, performance with onehot encoding, the composition of test sets, formal charges table, mutation category-wise performance statistics on S767, and statistics on transitive data augmentation are available in supporting information document.

\section{References}

(1) Serpente, N. Beyond a pedagogical tool: 30 years of Molecular Biology of the Cell. Nature Reviews Molecular Cell Biology 2013, 14, 120-125.

(2) Brannigan, J. A.; Wilkinson, A. J. Protein engineering 20 years on. Nature Reviews Molecular Cell Biology 2002, 3, 964-970. 
(3) Shaw, W. V. Protein engineering. The design, synthesis and characterization of factitious proteins. Biochemical Journal 1987, 246, 1-17.

(4) Zanghellini, A. de novo computational enzyme design. Current Opinion in Biotechnology 2014, 29, 132-138, Cell and Pathway Engineering.

(5) Erb, T. J.; Jones, P. R.; Bar-Even, A. Synthetic metabolism: metabolic engineering meets enzyme design. Current Opinion in Chemical Biology 2017, 37, 56-62, Biocatalysis and biotransformation, Bioinorganic Chemistry.

(6) Koellhoffer, J. F.; Higgins, C. D.; Lai, J. R. Protein engineering strategies for the development of viral vaccines and immunotherapeutics. FEBS Letters 2014, 588, 298307.

(7) Austin, H. P. et al. Characterization and engineering of a plastic-degrading aromatic polyesterase. Proceedings of the National Academy of Sciences 2018, 115, E4350E4357.

(8) Poluri, K. M.; Gulati, K. Protein Engineering Techniques: Gateways to Synthetic Protein Universe; Springer Singapore: Singapore, 2017; pp 103-134.

(9) Griss, R.; Schena, A.; Reymond, L.; Patiny, L.; Werner, D.; Tinberg, C. E.; Baker, D.; Johnsson, K. Bioluminescent sensor proteins for point-of-care therapeutic drug monitoring. Nat. Chem. Biol. 2014, 10, 598-603.

(10) Zhou, L.; Bosscher, M.; Zhang, C.; Özçubukçu, S.; Zhang, L.; Zhang, W.; Li, C. J.; Liu, J.; Jensen, M. P.; Lai, L., et al. A protein engineered to bind uranyl selectively and with femtomolar affinity. Nat. Chem. 2014, 6, 236-241.

(11) Raghunathan, G.; Sokalingam, S.; Soundrarajan, N.; Madan, B.; Munussami, G.; Lee, S.-G. Modulation of protein stability and aggregation properties by surface charge engineering. Molecular BioSystems 2013, 9, 2379-2389. 
(12) Coelho, M. B.; Ascher, D. B.; Gooding, C.; Lang, E.; Maude, H.; Turner, D.; Llorian, M.; Pires, D. E.; Attig, J.; Smith, C. W. Functional interactions between polypyrimidine tract binding protein and PRI peptide ligand containing proteins. Biochemical Society Transactions 2016, 44, 1058-1065.

(13) Siedhoff, N. E.; Schwaneberg, U.; Davari, M. D. In Enzyme Engineering and Evolution: General Methods; Tawfik, D. S., Ed.; Methods in Enzymology; Academic Press, 2020; Vol. 643; pp 281-315.

(14) Basheer, S. M.; Chellappan, S. Bioresources and bioprocess in biotechnology; Springer, 2017; pp 151-168.

(15) Yang, H.; Liu, L.; Li, J.; Chen, J.; Du, G. Rational Design to Improve Protein Thermostability: Recent Advances and Prospects. ChemBioEng Reviews 2015, 2, 87-94.

(16) van den Berg, B. A.; Reinders, M. J.; van der Laan, J.-M.; Roubos, J. A.; de Ridder, D. Protein redesign by learning from data. Protein Engineering, Design and Selection 2014, 27, 281-288.

(17) .Newton, M. S.; Arcus, V. L.; Gerth, M. L.; Patrick, W. M. Enzyme evolution: innovation is easy, optimization is complicated. Current Opinion in Structural Biology 2018, 48, 110-116, Folding and binding in silico, in vitro and in cellula • Proteins: An Evolutionary Perspective.

(18) Robertson, A. D.; Murphy, K. P. Protein Structure and the Energetics of Protein Stability. Chemical Reviews 1997, 97, 1251-1268.

(19) Modarres, H. P.; Mofrad, M. R.; Sanati-Nezhad, A. Protein thermostability engineering. RSC Adv. 2016, 6, 115252-115270.

(20) Goldenzweig, A.; Fleishman, S. J. Principles of Protein Stability and Their Application 
in Computational Design. Annual Review of Biochemistry 2018, 87, 105-129, PMID: 29401000.

(21) Tokuriki, N.; Stricher, F.; Serrano, L.; Tawfik, D. S. How Protein Stability and New Functions Trade Off. PLOS Computational Biology 2008, 4, 1-7.

(22) Wang, Y.; Yu, X.; Zhao, H. Biosystems design by directed evolution. AIChE Journal 2020, 66, e16716.

(23) Gill, M.; McCully, M. E. Molecular dynamics simulations suggest stabilizing mutations in a de novo designed $\alpha / \beta$ protein. Protein Engineering, Design and Selection 2020, 32, 317-329.

(24) Chen, Q.; Xiao, Y.; Shakhnovich, E. I.; Zhang, W.; Mu, W. Semi-rational design and molecular dynamics simulations study of the thermostability enhancement of cellobiose 2-epimerases. International Journal of Biological Macromolecules 2020, 154, 13561365.

(25) Chen, Y.; Lu, H.; Zhang, N.; Zhu, Z.; Wang, S.; Li, M. PremPS: Predicting the impact of missense mutations on protein stability. PLOS Computational Biology 2021, 16, $1-22$.

(26) Frenz, B.; Lewis, S. M.; King, I.; DiMaio, F.; Park, H.; Song, Y. Prediction of Protein Mutational Free Energy: Benchmark and Sampling Improvements Increase Classification Accuracy. Frontiers in Bioengineering and Biotechnology 2020, 8, 1175.

(27) Li, B.; Yang, Y. T.; Capra, J. A.; Gerstein, M. B. Predicting changes in protein thermostability upon point mutation with deep 3D convolutional neural networks. bioRxiv 2020 ,

(28) Montanucci, L.; Capriotti, E.; Frank, Y.; Ben-Tal, N.; Fariselli, P. DDGun: an un- 
trained method for the prediction of protein stability changes upon single and multiple point variations. BMC Bioinformatics 2019, 20, 335.

(29) Dehouck, Y.; Kwasigroch, J. M.; Gilis, D.; Rooman, M. PoPMuSiC 2.1: a web server for the estimation of protein stability changes upon mutation and sequence optimality. BMC Bioinformatics 2011, 12, 151.

(30) Schymkowitz, J.; Borg, J.; Stricher, F.; Nys, R.; Rousseau, F.; Serrano, L. The FoldX web server: an online force field. Nucleic acids research 2005, 33, W382-W388.

(31) Dehouck, Y.; Grosfils, A.; Folch, B.; Gilis, D.; Bogaerts, P.; Rooman, M. Fast and accurate predictions of protein stability changes upon mutations using statistical potentials and neural networks: PoPMuSiC-2.0. Bioinformatics 2009, 25, 2537-2543.

(32) Fariselli, P.; Martelli, P. L.; Savojardo, C.; Casadio, R. INPS: predicting the impact of non-synonymous variations on protein stability from sequence. Bioinformatics 2015, 31, 2816-2821.

(33) Savojardo, C.; Fariselli, P.; Martelli, P. L.; Casadio, R. INPS-MD: a web server to predict stability of protein variants from sequence and structure. Bioinformatics $\mathbf{2 0 1 6}$, 32, 2542-2544.

(34) Laimer, J.; Hofer, H.; Fritz, M.; Wegenkittl, S.; Lackner, P. MAESTRO - multi agent stability prediction upon point mutations. BMC Bioinformatics 2015, 16, 116.

(35) Worth, C. L.; Preissner, R.; Blundell, T. L. SDM-a server for predicting effects of mutations on protein stability and malfunction. Nucleic acids research $\mathbf{2 0 1 1}$, 39, W215W222.

(36) Pires, D. E. V.; Ascher, D. B.; Blundell, T. L. mCSM: predicting the effects of mutations in proteins using graph-based signatures. Bioinformatics 2013, 30, 335-342. 
(37) Pires, D. E.; Ascher, D. B.; Blundell, T. L. DUET: a server for predicting effects of mutations on protein stability using an integrated computational approach. Nucleic Acids Research 2014, 42, W314-W319.

(38) Rodrigues, C. H.; Pires, D. E.; Ascher, D. B. DynaMut2: Assessing changes in stability and flexibility upon single and multiple point missense mutations. Protein Science 2021, 30, 60-69.

(39) Kulandaisamy, A.; Zaucha, J.; Frishman, D.; Gromiha, M. M. MPTherm-pred: Analysis and Prediction of Thermal Stability Changes upon Mutations in Transmembrane Proteins. Journal of Molecular Biology 2020, 166646.

(40) Huang, P.; Chu, S. K. S.; Frizzo, H. N.; Connolly, M. P.; Caster, R. W.; Siegel, J. B. Evaluating Protein Engineering Thermostability Prediction Tools Using an Independently Generated Dataset. ACS Omega 2020, 5, 6487-6493.

(41) Wales, D. Energy landscapes: Applications to clusters, biomolecules and glasses; Cambridge University Press, 2003.

(42) Raghunathan, S.; Jaganade, T.; Priyakumar, U. D. Urea-aromatic interactions in biology. Biophys. Rev. 2020, 12, 65-84.

(43) Sanavia, T.; Birolo, G.; Montanucci, L.; Turina, P.; Capriotti, E.; Fariselli, P. Limitations and challenges in protein stability prediction upon genome variations: towards future applications in precision medicine. Computational and structural biotechnology journal 2020, 18, 1968-1979.

(44) Fang, J. A critical review of five machine learning-based algorithms for predicting protein stability changes upon mutation. Briefings in Bioinformatics 2019, 21, 1285-1292.

(45) Pucci, F.; Bernaerts, K. V.; Kwasigroch, J. M.; Rooman, M. Quantification of biases 
in predictions of protein stability changes upon mutations. Bioinformatics 2018, 34, 3659-3665.

(46) Thiltgen, G.; Goldstein, R. A. Assessing Predictors of Changes in Protein Stability upon Mutation Using Self-Consistency. PLOS ONE 2012, 7, 1-6.

(47) Benedix, A.; Becker, C. M.; de Groot, B. L.; Caflisch, A.; Böckmann, R. A. Predicting free energy changes using structural ensembles. Nature Methods 2009, 6, 3-4.

(48) Pandurangan, A. P.; Ochoa-Montaño, B.; Ascher, D. B.; Blundell, T. L. SDM: a server for predicting effects of mutations on protein stability. Nucleic Acids Research 2017, 45, W229-W235.

(49) Cao, H.; Wang, J.; He, L.; Qi, Y.; Zhang, J. Z. DeepDDG: Predicting the Stability Change of Protein Point Mutations Using Neural Networks. Journal of Chemical Information and Modeling 2019, 59, 1508-1514.

(50) Benevenuta, S.; Pancotti, C.; Fariselli, P.; Birolo, G.; Sanavia, T. An antisymmetric neural network to predict free energy changes in protein variants. Journal of Physics D: Applied Physics 2021, 54, 245403.

(51) Pathak, Y.; Mehta, S.; Priyakumar, U. D. Learning Atomic Interactions through Solvation Free Energy Prediction Using Graph Neural Networks. Journal of Chemical Information and Modeling 2021, 61, 689-698.

(52) Laghuvarapu, S.; Pathak, Y.; Priyakumar, U. D. BAND NN: A Deep Learning Framework for Energy Prediction and Geometry Optimization of Organic Small Molecules. Journal of Computational Chemistry 2020, 41, 790-799.

(53) Aggarwal, R.; Gupta, A.; Chelur, V.; Jawahar, C. V.; Priyakumar, U. D. DeepPocket: Ligand Binding Site Detection and Segmentation using 3D Convolutional Neural Networks. 2021, 
(54) Senior, A. W. et al. Improved protein structure prediction using potentials from deep learning. Nature 2020, 577, 706-710.

(55) Bagal, V.; Aggarwal, R.; Vinod, P. K.; Priyakumar, U. D. LigGPT: Molecular Generation using a Transformer-Decoder Model. 2021,

(56) Yang, J.; Anishchenko, I.; Park, H.; Peng, Z.; Ovchinnikov, S.; Baker, D. Improved protein structure prediction using predicted interresidue orientations. Proceedings of the National Academy of Sciences 2020, 117, 1496-1503.

(57) Caldararu, O.; Mehra, R.; Blundell, T. L.; Kepp, K. P. Systematic Investigation of the Data Set Dependency of Protein Stability Predictors. Journal of Chemical Information and Modeling 2020, 60, 4772-4784, PMID: 32786698.

(58) Pucci, F.; Bernaerts, K.; Teheux, F.; Gilis, D.; Rooman, M. Symmetry Principles in Optimization Problems: an application to Protein Stability Prediction. IFACPapersOnLine 2015, 48, 458-463, 8th Vienna International Conferenceon Mathematical Modelling.

(59) Stourac, J.; Dubrava, J.; Musil, M.; Horackova, J.; Damborsky, J.; Mazurenko, S.; Bednar, D. FireProtDB: database of manually curated protein stability data. Nucleic Acids Research 2020, 49, D319-D324.

(60) Kabsch, W.; Sander, C. Dictionary of protein secondary structure: Pattern recognition of hydrogen-bonded and geometrical features. Biopolymers 1983, 22, 2577-2637.

(61) Touw, W. G.; Baakman, C.; Black, J.; te Beek, T. A.; Krieger, E.; Joosten, R. P.; Vriend, G. A series of PDB-related databanks for everyday needs. Nucleic Acids Research 2014, 43, D364-D368.

(62) Kawashima, S.; Kanehisa, M. AAindex: Amino Acid index database. Nucleic Acids Research 2000, 28, 374-374. 
(63) Mazurenko, S. Predicting protein stability and solubility changes upon mutations: data perspective. ChemCatChem 2020, 12, 5590-5598.

(64) Cock, P. J. A.; Antao, T.; Chang, J. T.; Chapman, B. A.; Cox, C. J.; Dalke, A.; Friedberg, I.; Hamelryck, T.; Kauff, F.; Wilczynski, B.; de Hoon, M. J. L. Biopython: freely available Python tools for computational molecular biology and bioinformatics. Bioinformatics 2009, 25, 1422-1423.

(65) Konopka, B. M.; Marciniak, M.; Dyrka, W. Quantiprot - a Python package for quantitative analysis of protein sequences. BMC Bioinformatics 2017, 18, 339 .

(66) Paszke, A. et al. In Advances in Neural Information Processing Systems 32; Wallach, H., Larochelle, H., Beygelzimer, A., d'Alché-Buc, F., Fox, E., Garnett, R., Eds.; Curran Associates, Inc., 2019; pp 8024-8035.

(67) Pedregosa, F. et al. Scikit-learn: Machine Learning in Python. Journal of Machine Learning Research 2011, 12, 2825-2830.

(68) Harris, C. R. et al. Array programming with NumPy. Nature 2020, 585, 357-362.

(69) Barron, J. T. A More General Robust Loss Function. CoRR 2017, abs/1701.030r7.

(70) Kingma, D. P.; Ba, J. Adam: A Method for Stochastic Optimization. 2017.

(71) Kwasigroch, J. M.; Gilis, D.; Dehouck, Y.; Rooman, M. PoPMuSiC, rationally designing point mutations in protein structures. Bioinformatics 2002, 18, 1701-1702.

(72) Masso, M.; Vaisman, I. I. AUTO-MUTE: web-based tools for predicting stability changes in proteins due to single amino acid replacements. Protein Engineering, Design and Selection 2010, 23, 683-687.

(73) Parthiban, V.; Gromiha, M. M.; Schomburg, D. CUPSAT: prediction of protein stability upon point mutations. Nucleic acids research 2006, 34, W239-W242. 
(74) Usmanova, D. R.; Bogatyreva, N. S.; Ariño Bernad, J.; Eremina, A. A.; Gorshkova, A. A.; Kanevskiy, G. M.; Lonishin, L. R.; Meister, A. V.; Yakupova, A. G.; Kondrashov, F. A.; Ivankov, D. N. Self-consistency test reveals systematic bias in programs for prediction change of stability upon mutation. Bioinformatics 2018, 34, 3653-3658.

(75) Cheng, J.; Randall, A.; Baldi, P. Prediction of protein stability changes for single-site mutations using support vector machines. Proteins: Structure, Function, and Bioinformatics 2006, 62, 1125-1132.

(76) Pathak, Y.; Laghuvarapu, S.; Mehta, S.; Priyakumar, U. D. Chemically Interpretable Graph Interaction Network for Prediction of Pharmacokinetic Properties of Drug-Like Molecules. Proceedings of the AAAI Conference on Artificial Intelligence 2020, 34, 873-880.

(77) Yin, S.; Ding, F.; Dokholyan, N. V. Modeling Backbone Flexibility Improves Protein Stability Estimation. Structure 2007, 15, 1567-1576.

(78) Kellogg, E. H.; Leaver-Fay, A.; Baker, D. Role of conformational sampling in computing mutation-induced changes in protein structure and stability. Proteins 2011, 79, 830838, 21287615[pmid]. 


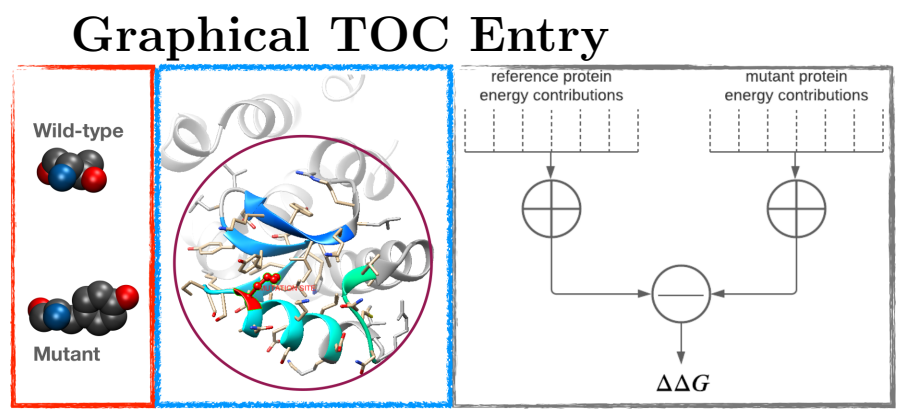




\title{
Supporting Information:
}

\section{SCONES: Self-Consistent Neural Network for Protein Stability Prediction Upon Mutation}

\author{
Yashas Samaga B L, Shampa Raghunathan, and U. Deva Priyakumar* \\ Center for Computational Natural Sciences and Bioinformatics, International Institute of \\ Information Technology, Hyderabad 500 032, India \\ E-mail: deva@iiit.ac.in
}

\section{Transitive Data Augmentation}

Table S1: FireProtDB sizes after data augmentation FireProtDB is transitively closed for single-point forward mutations. However, computing transitive closure after adding hypothetical reverse mutations generates a lot of new transitive single-point mutation samples. "cleaned" dataset has inconsistent samples removed as outlined in the main text. "unique" indicates duplicates have been merged as mentioned in the main text. "symmetric" indicates the addition of hypothetical reverse mutations and "transitive" indicates transitive closure for single-point mutations. Note that we do not use transitively augmented datasets in our work.

\begin{tabular}{l|l}
\hline Dataset & number of samples (including duplicates) \\
\hline fireprotdb & 17118 \\
fireprotdb_cleaned & 17092 \\
fireprotdb_cleaned_unique & 8628 \\
fireprotdb_cleaned_unique_symmetric & 17251 \\
fireprotdb_cleaned_unique_symmetric_transitive & 49081 \\
\hline
\end{tabular}

The samples in these datasets include both $\Delta \Delta G$ and $\Delta T_{m}$ samples. FireProtDB web portal indicates that there are 15989 experiments as of February 25th. The CSV dump downloaded from their web portal contains 17118 entries. There are some exact duplicates that might explain the difference between the two numbers. 
In our transitive data augmentation procedure, we compute the transitive closure on the symmetrically closed dataset without duplicates. Any new duplicate samples that arise during transitive data augmentation are rejected. It might be beneficial to retain the duplicates and then merge duplicates afterward. We also fix positions during transitive data augmentation to avoid generating samples with multiple mutations. Methods trained for predicting stability for multiple mutations might greatly benefit from large increases in dataset size without the position restriction.

$\Delta \Delta G$ of the transitive samples are exact if the uncertainties in $\Delta \Delta G_{Y \rightarrow Z}$ and $\Delta \Delta G_{X \rightarrow Y}$ values are zero. In principle, if the uncertainty in each $\Delta \Delta G$ value is $\sigma$, the uncertainty in the transitive $\Delta \Delta G$ calculated will be $2 \sigma$. However, if the $\Delta G_{Y}$ terms involved in both $\Delta \Delta G_{Y \rightarrow Z}$ and $\Delta \Delta G_{X \rightarrow Y}$ are identical, the uncertainty would be $\sigma$ as the errors in $\Delta G_{Y}$ would cancel out, and it is effectively equivalent to calculating $\Delta \Delta G$ directly from $\Delta G_{X}$ and $\Delta G_{Z}$

\subsection{Are transitive samples useful for architectures that incorpo- rate transitive property?}

Transitive data augmentation is definitely useful for architectures that do not incorporate transitive consistency into the architecture. But they are also useful for architectures that incorporate the property. Consider a model that incorporates transitive consistency into the architecture. Let the errors in predictions for the three samples $\Delta \Delta G_{X \rightarrow Y}, \Delta \Delta G_{Y \rightarrow Z}$ and $\Delta \Delta G_{X \rightarrow Z}$ be denoted by $\Delta_{1}, \Delta_{2}$ and $\Delta_{3}$ respectively. It is possible that $\Delta_{1}$ and $\Delta_{2}$ cancel out to give a near-zero $\Delta_{3}$. This shows that a transitive sample can result in a very different loss compared to the losses of the constituent samples. It is also possible that the original dataset contains two samples that have low errors, but their corresponding new transitive sample has a high error. Therefore, augmenting datasets with transitive samples is not redundant. 


\section{Performance with embedding layer}

The fixed amino acid features was replaced with an embedding layer outputting the same number of features. The statistics here must be compared with the results section of the manuscript.

Table S2: Performance on different test sets

\begin{tabular}{|l|lll|ll|ll|}
\hline & & & & \multicolumn{2}{|l|}{ stabilization } & \multicolumn{2}{|c|}{ destabilization } \\
\cline { 5 - 8 } Dataset & PCC & MAE & RMSE & AUC $^{\mathrm{a}}$ & PCC $^{\mathrm{b}}$ & $\mathrm{AUC}^{\mathrm{a}}$ & PCC $^{\mathrm{b}}$ \\
\hline S350 & 0.55 & 1.13 & 1.52 & 0.81 & 0.63 & 0.72 & 0.43 \\
S350 (hard) & 0.52 & 1.17 & 1.54 & 0.76 & 0.52 & 0.73 & 0.44 \\
S $^{\text {sym }}$ & 0.55 & 1.08 & 1.55 & 0.76 & 0.38 & 0.77 & 0.46 \\
S $^{\text {transitive }}$ & 0.55 & 1.08 & 1.55 & 0.76 & 0.38 & 0.77 & 0.46 \\
S $^{\text {sym }}$ (hard) & 0.50 & 1.15 & 1.61 & 0.71 & 0.35 & 0.72 & 0.39 \\
S767 & 0.43 & 1.28 & 1.94 & 0.69 & 0.31 & 0.68 & 0.38 \\
S767 (hard) & 0.26 & 1.43 & 2.11 & 0.54 & -0.10 & 0.60 & 0.27 \\
\hline
\end{tabular}

RMSE and MAE are in $\mathrm{kcal} / \mathrm{mol}$.

${ }^{a}$ AUC is calculated for strongly affecting mutations only $(|\Delta \Delta G| \geq 1$ $\mathrm{kcal} / \mathrm{mol}$ )

${ }^{\mathrm{b}}$ PCC is calculated using all the samples on either side of zero

Table S3: Performance on $\mathbf{S}^{\text {sym }}$

\begin{tabular}{|l|l|l|l|l|l|l|l|}
\hline \multirow{2}{*}{\multicolumn{1}{|c|}{ Method }} & \multicolumn{2}{|c|}{ forward } & \multicolumn{2}{c|}{ reverse } & & & \\
\cline { 2 - 8 } & PCC & RMSE & PCC & RMSE & $R_{F R}$ & $\langle\delta\rangle$ & $\Delta$ \\
\hline SCONES & 0.51 & 1.51 & 0.47 & 1.57 & -0.95 & 0.07 & 0.34 \\
SCONES (Stransitive $)$ & 0.51 & 1.52 & 0.47 & 1.57 & -0.96 & 0.06 & 0.32 \\
SCONES (hard) & 0.44 & 1.61 & 0.43 & 1.61 & -0.99 & 0.00 & 0.13 \\
\hline
\end{tabular}

RMSE, $\langle\delta\rangle$ and $\Delta$ are in $\mathrm{kcal} / \mathrm{mol}$.

Table S4: Transitive consistency on $\mathbf{S}^{\text {transitive }}$.

\begin{tabular}{|c|c|c|}
\hline Method & $R_{T}$ & $\Delta_{T}$ \\
\hline SCONES $\left(\mathbf{S}^{\text {sym }}\right)$ & 0.98 & 0.21 \\
\hline SCONES $\left(\mathbf{S}^{\text {transitive }}\right)$ & 0.98 & 0.21 \\
\hline SCONES ( $\mathbf{S}^{\mathrm{sym}}$ hard) & 0.99 & 0.13 \\
\hline
\end{tabular}

$\Delta_{T}$ is in $\mathrm{kcal} / \mathrm{mol}$. 


\section{$3 \quad$ Neural network architecture and training}

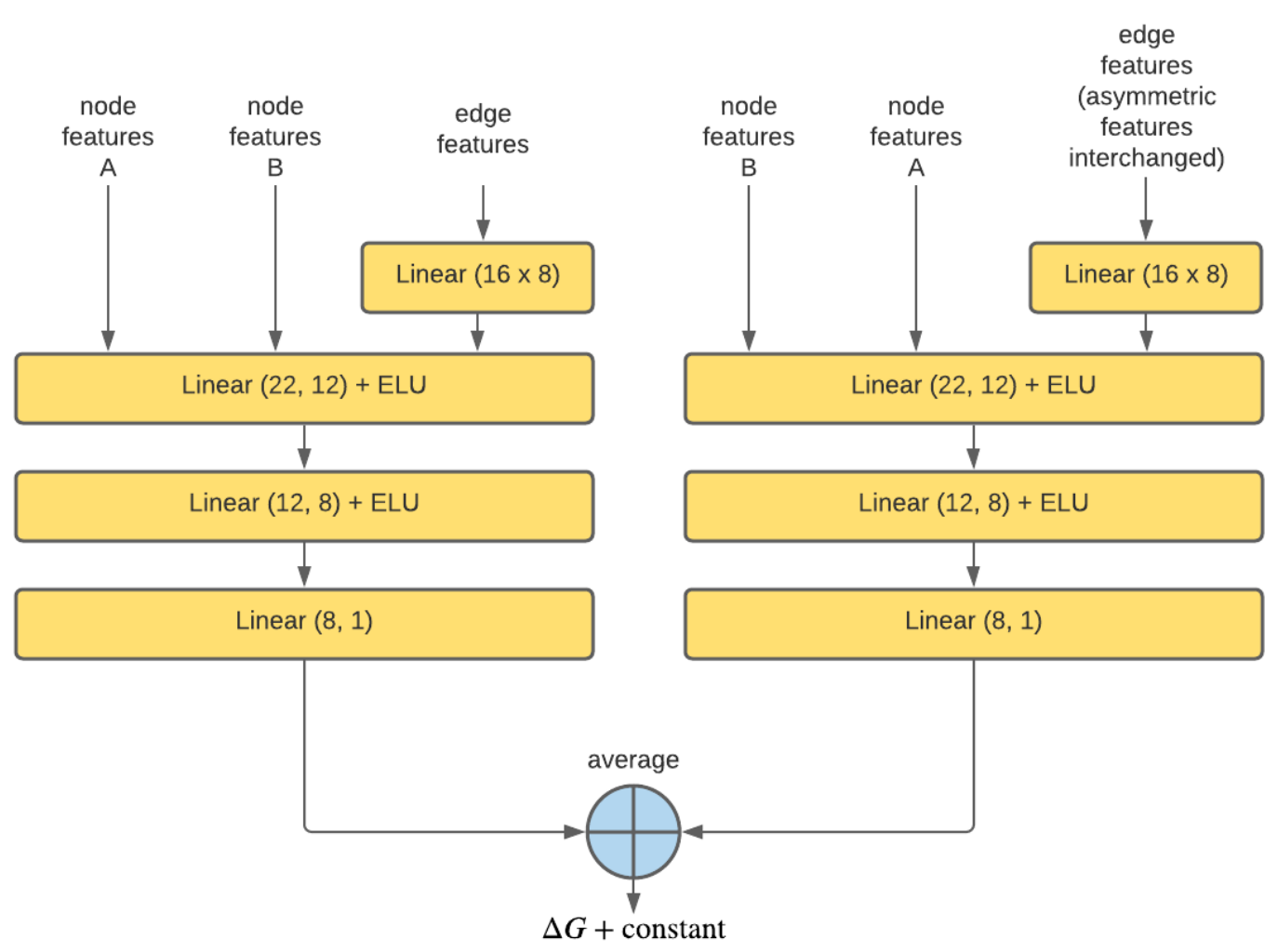

Figure S1: The contributions of residues A and B must be the same irrespective of which residue features appear first in the feature vector. This constraint is enforced by passing two feature vectors in parallel through the network (like Siamese networks) as depicted in the picture. The network contains 526 trainable parameters.

Adam optimizer was used for training. The initial learning rate was 0.002. 0.9 and 0.999 were the beta coefficients used for decaying the running average of the gradient and its square. $10^{-8}$ was set as epsilon. $10^{-5}$ was used for weight decay. The networks were trained for 40 epochs with ReduceLROnPlateau LR scheduler with factor as 0.1, patience as 8, cooldown as 5, min_lr as $10^{-5}$ and threshold as $10^{-3}$. 


\section{Formal charge table}

Table S5: Formal charges

\begin{tabular}{l|l}
\hline Amino acid code & charge \\
\hline A & 0 \\
C & 0 \\
D & -1 \\
E & -1 \\
F & 0 \\
G & 0 \\
H & 1 \\
I & 0 \\
K & 1 \\
L & 0 \\
M & 0 \\
N & 0 \\
P & 0 \\
Q & 1 \\
R & 1 \\
S & 0 \\
T & 0 \\
V & 0 \\
W & 0 \\
Y & 0 \\
\hline
\end{tabular}




\section{Composition of test sets}

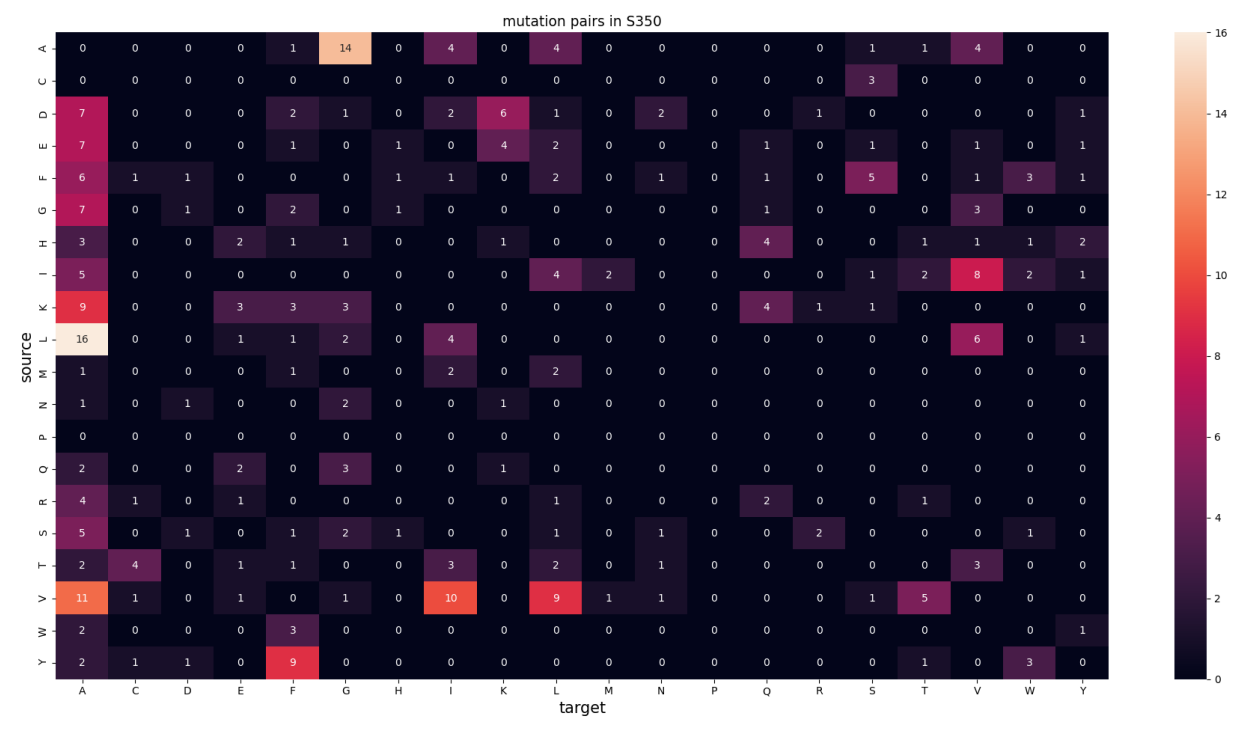

Figure S2: Mutation type confusion matrix for S350

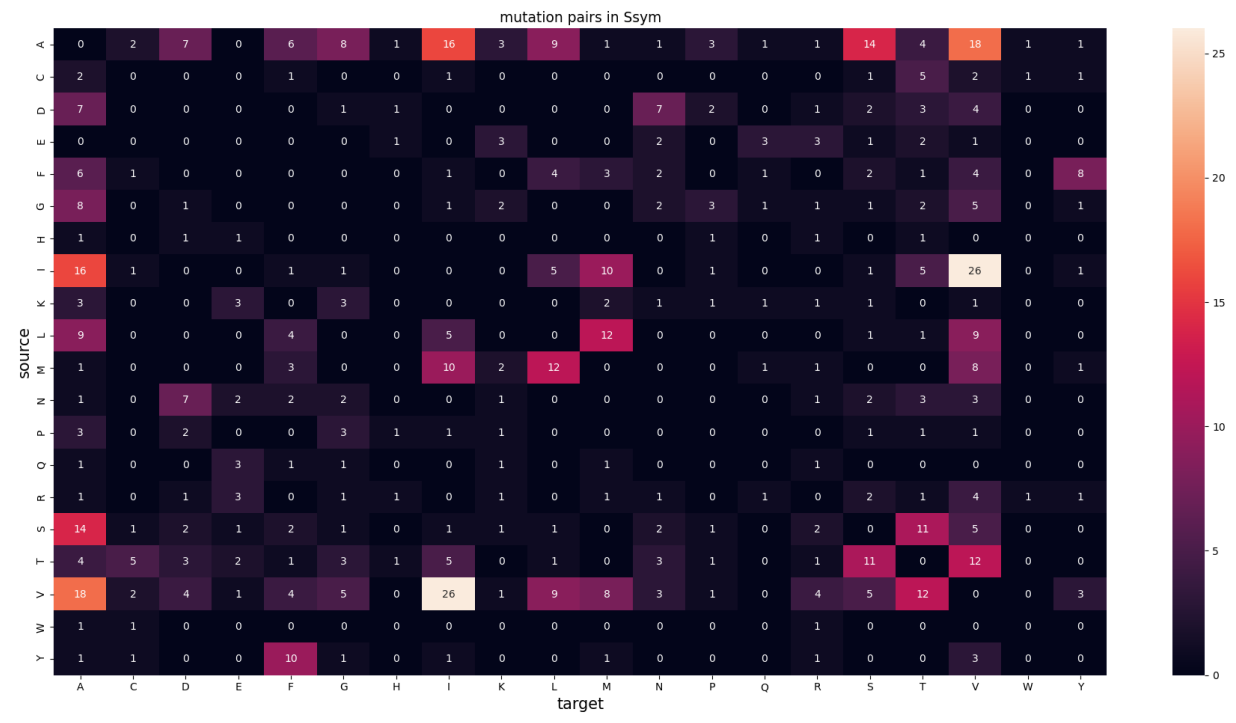

Figure S3: Mutation type confusion matrix for in $\mathrm{S}^{\mathrm{sym}}$ 


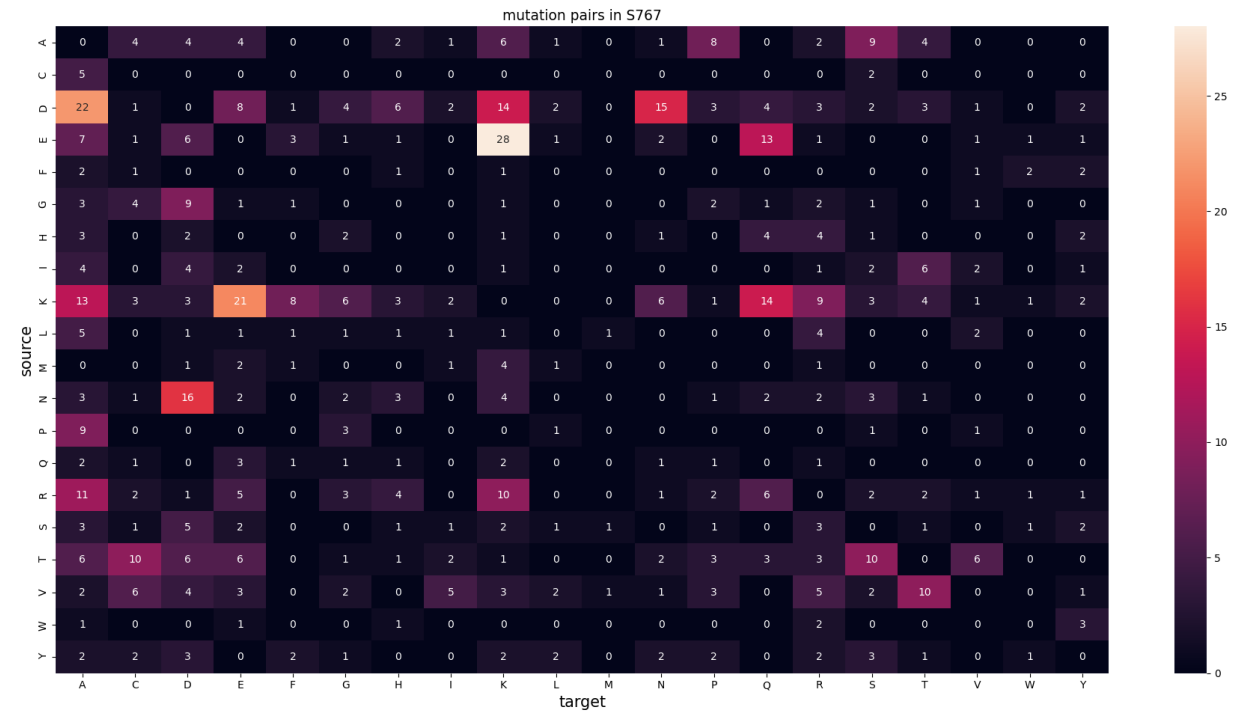

Figure S4: Mutation type confusion matrix for in S767 


\section{Category-wise evaluation on S767}

Table S6 provides category-wise performance of our method on S767 dataset. We note that the performance varies significantly across categories. Mutations in some categories are intrinsically hard to learn while some are easy but a method might fail to learn well. A part of this variation could be because of the composition of the training sets. Protein thermodynamics databases are largely derived from literature search which are biased by human selection and common procedures such as alanine scanning. Our training sets were directly derived from protein thermodynamics databases without a sampling process that would balance the training set. Thus, the trained models might have inherited the biases of the protein thermodynamics databases. Improved sampling procedures for training sets might help mitigate variations in category-wise performance. Our model performs badly on mutations involving proline and small-to-large mutations. The small-to-large issues can migrate to other categories like hydrophobic to positive (all positively charged residues are bulky). Mutations involving proline often disrupt the protein structure significantly and are hard to predict. Small-to-large mutations frequently cause steric clashes that lead to structural rearrangements. ${ }^{\mathrm{S} 1}$ Many methods relax the structure ${ }^{\mathrm{S} 2-\mathrm{S} 5}$ to accommodate small structural changes which improves the performance. We also note the high variation in

performance metrics in each category. This variation suggests that the model performance in some categories significantly depends on the initialization. 


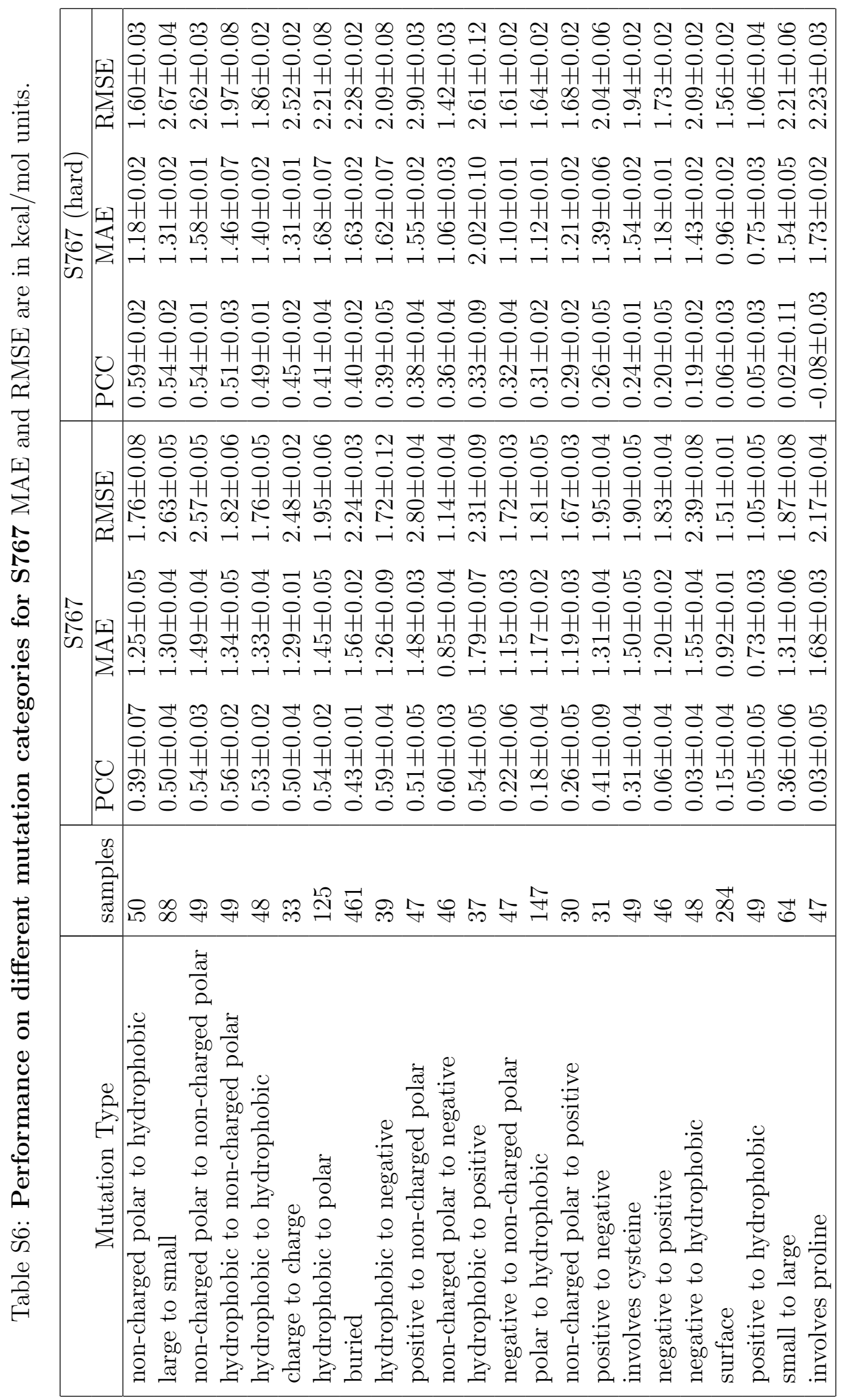




\section{References}

(S1) Yin, S.; Ding, F.; Dokholyan, N. V. Modeling Backbone Flexibility Improves Protein Stability Estimation. Structure 2007, 15, 1567-1576.

(S2) Chen, Y.; Lu, H.; Zhang, N.; Zhu, Z.; Wang, S.; Li, M. PremPS: Predicting the impact of missense mutations on protein stability. PLOS Computational Biology 2021, 16, 1-22.

(S3) Li, B.; Yang, Y. T.; Capra, J. A.; Gerstein, M. B. Predicting changes in protein thermostability upon point mutation with deep 3D convolutional neural networks. bioRxiv 2020 ,

(S4) Schymkowitz, J.; Borg, J.; Stricher, F.; Nys, R.; Rousseau, F.; Serrano, L. The FoldX web server: an online force field. Nucleic acids research 2005, 33, W382-W388.

(S5) Kellogg, E. H.; Leaver-Fay, A.; Baker, D. Role of conformational sampling in computing mutation-induced changes in protein structure and stability. Proteins 2011, 79, 830$838,21287615[\mathrm{pmid}]$. 
